\title{
Towards Future Installations: Mutual Interactions of Short Intakes with Modern High Bypass Fans
}

\author{
Nagabhushana Rao Vadlamani * \\ Department of Aerospace Engineering \\ Indian Institute of Technology, Madras \\ Chennai, India, 600036 \\ Email: nrv@iitm.ac.in \\ Teng Cao \\ Whittle Laboratory \\ Department of Engineering \\ University of Cambridge \\ Cambridge, UK, CB2 1PZ \\ Email: tc367@cam.ac.uk
}

\author{
Rob Watson \\ Ashby Building \\ Queen's University Belfast, \\ Northern Ireland, UK, BT9 5AH \\ Email: R.Watson@qub.ac.uk \\ Paul G. Tucker \\ CFD Laboratory \\ Department of Engineering \\ University of Cambridge \\ Cambridge, UK, CB2 1PZ \\ Email: pgt23@cam.ac.uk
}

School of Mechanical and Aerospace Engineering

In this paper, we investigate the coupled interaction between a new short intake design with a modern fan in a high-bypass ratio civil engine, specifically under the off-design condition of high incidence. The interaction is expected to be much more significant than that on a conventional intake. The performance of both the intake-alone and rotor-alone configurations are examined under isolation. Subsequently, a comprehensive understanding on the two-way interaction between intake and fan is presented. This includes the effect of fan on intake AoA tolerance (FoI) and the effect of circumferential and radial flow distortion induced by the intake on the fan-performance (IoF). In FoI scenario, the rotor effectively redistributes the mass flow at the fan-face. The AoA tolerance of the short-intake design has increased by $\approx 4^{\circ}$ when compared to the intake-alone configuration. Dynamic nature of distortion due to shock-unsteadiness has been quantified.

\footnotetext{
*Address all correspondence for other issues to this author.
}

Space-time (ST) plots and power spectral density (PSD) of pressure fluctuations show the existence of a spectral gap between the shock unsteadiness and blade passing, with almost an order of magnitude difference in the corresponding frequencies. In IoF scenario, both the 'large' $\left(\mathrm{O}\left(360^{\circ}\right)\right)$ and 'small' scale distortion $\left(O\left(10-60^{\circ}\right)\right)$ induced by the intake results in a non-uniform inflow to the rotor. Sector analysis reveals a substantial variation in the local operating condition of the fan as opposed to its steady characteristic. Streamline curvature, upwash and wake thickening are identified to be the three key factors affecting the fan performance. These underlying mechanisms are discussed in detail to provide further insights into the physical understanding of the fan-intake interaction. In addition to the shock induced separation on the intake lip, the current study shows that shorter intakes are much more prone to the upwash effect at higher AoA. Insufficient flow straightening along the engine axis is reconfirmed to be one of the limiting factors 
for the short-intake design.

\section{Introduction}

The aviation industry is committed to developing efficient, quiet and environmentally friendly aircraft engines. In this quest, there is an increasing trend in the gas turbine industry to shift towards the turbofan engines with higher bypass ratios (BPR) and lower fan pressure ratios (FPR). Such modern architectures offer the fuel burn benefits and reduce the emissions and noise $[1,2]$. With increasing BPR, the diameter of the engine inlet increases. However, powerplant weight and nacelle aerodynamic interaction with the aircraft wing have to remain within acceptable limits. This imposes a design constraint on relatively shorter intakes and nacelles with much more severe restrictions on external diameter. These geometric constraints have the consequential effect of limiting the use of current conventional intake lip and diffuser shapes needed for full flight envelope capability within currently accepted separation limits for fan-intake compatibility $[3,4]$. As a result, much more comprehensive understanding and computational ability to predict fan to intake aerodynamic interactions are required to enable future viable intake designs.

An optimal intake design is the one which provides a uniform distribution of the air flow with minimum total pressure loss over a wide range of operating conditions. However, shorter intakes can result in a reduced incidence tolerance and the flow is much more prone to separation, specifically under off-design conditions like high incidence/crosswinds. There is also an increased aerodynamic interaction between the downstream fan and the distorted flow over the intake under off-design conditions. This can subsequently reduce the stability margin of the fan $[5,6]$ and can ultimately lead to adverse interactions like stall [7]. The overall performance of the propulsion system is sensitive to this interaction of the separated flow with the fan. Figure 1(a) shows the key flow features of intake operating under high incidence.

Future propulsion and aircraft concepts, such as ultrahigh-bypass-ratio engine and boundary layer ingestion (BLI) aircraft have received considerable attention over the last decade. In these systems, installation effects have become increasingly challenging as the fan is presented with distortions of total pressure, static pressure, and flow angle. Several studies in literature addressed the effect of distortion on the fan-performance and vice versa. In the context of intakes subjected to high-incidence, Makuni et al. [8] and Coschignano et al. [9] built an experimental rig based on the highly loaded two-dimensional section of the intake lip. They explored the dynamics of the shock-wave boundary layer interaction (SWBLI) and the three-dimensional flow field with increasing incidence onto the intake. Experiments by Hodder [10] and Larkin and Schweiger [11] and computations by Peters et al. [3], Cao et al. [12] and Carnevale et al. [13] studied the effect of downstream fan on the 'incidence tolerance' of an intake i.e. the angle of attack at which the flow separates over an intake lip. In these computational studies, the effect of fan is modelled either using body force models or similarity models. In all these studies, it has been noted that the fan alleviates the post-separation distortion transferred to the fan-face. Cao et al. [12] noted that the fan redistributes the incoming mass flow and effectively increases the separation-free angle of attack. An increase of $\approx 4^{\circ}$ has been reported when the proximity of the fan is closest to the intake lip. Peters et al. [3] identified a critical L/D of 0.25 under which the benefit offered by short intakes is outweighed by the fan-efficiency penalties. Two critical aspects: (a) interaction of the rotor with a region of high streamwise Mach number at the fan face and (b) reduced flow straightening through the inlet contribute to this drop in the fan performance. Peters [3] demonstrated the influence of the pylon's potential field to be most dominant during the cruise when compared to the off-design condition. Shielding the rotor from pylon using non-axisymmetric fan exit guide vanes is shown to improve the fan efficiency by $0.25 \%$. On a conventional intake-fan configuration, Carnevale et al. [14] report that the fan attenuates long wavelength components of the flow non-uniformity by a factor of five, while the small wavelengths either remained unattenuated or marginally amplified.

In the context of boundary layer ingesting (BLI) engines (in the absence of AoA), Fidalgo et al. [15] studied the fan-distortion interaction through both experiments complemented by unsteady computations. An incoming total pressure distortion spanning $120^{\circ}$ of the circumference induced both co-swirl and counter-swirl upstream of the fan. This is shown to greatly affect both the fan work input and its local operating condition. Novel post-processing tools are used to track the fan performance as it orbits through the distortion. Gunn et al. [16] carried out high resolution measurements of BLI engine to identify the loss sources within a low-speed fan stage subjected to a inlet total pressure distortion in a $60^{\circ}$ sector. They report a $4.6 \%$ drop in the fan rotor efficiency due to circumferential and radial variation of the incidence angle and a substantial flow separation at the rotor tip caused due to the high incidence in the distorted sector. Several studies were also carried out under the NASA BLI Inlet/Distortion fan program and NASA advanced air transport technology project. By means of CFD-based analysis, Ochs et al. [17] predicted a benefit of $4.2 \%$ in the propulsive efficiency for BLI propulsion system when compared to the conventional propulsion system. However, designing the rotor of a BLI system is a challenging task as the fan encounters severe aerodynamic and mechanical loads. Cousins [18] designed and tested a distortion-tolerant fan accounting for the highly coupled interaction between the inlet and the rotor. Frohnapfel et al. [19] have largely decoupled the total pressure and swirl distortion and measured the rotors response to each of these distortions. They have noted that the swirl distortion largely affects the flow angles at the fan-exit, while the pressure distortion significantly affects the total and static pressure ratio across the fan.

Cousins and Davis [20] extended the parallel compressor theory to include the (a) effects of swirl, (b) dynamic response of the compression system using a dynamic lag ra- 
tio, (c) circumferential mass redistribution and (d) meanline model into the dynamic parallel compressor code. In a companion paper [6], this dynamic modelling system has been applied to simulate a variety of complex distortion patterns including pressure, temperature and swirl distortions and favourably compared the predictions against the test data. In this two-part paper $[6,20]$, authors also report the difficulties to include the effect of radial mass redistribution into the extended parallel compressor model, hence suggesting the need for high fidelity simulations to quantify the radial distortion effects on system performance. Recent investigations by $\mathrm{Ma}$ et al. $[21,22]$ introduce a mixed fidelity approach to model fan-distortion interaction, where the separation downstream of the distortion generator is captured using high fidelity LES, while the fan is modelled using immersed boundary method. Subsequently, different parametric studies are carried out for varying proximities of the fan and distortion generator. Interestingly, with decreasing proximity, the inaccuracies of the turbulence model within the separated region are found to be mitigated by the strong acceleration due to the fan.

\section{Scope of the paper}

In this paper, we investigate the fan-intake interaction in a high-bypass ratio civil engine. A relatively shorter intake with a slimmer lip is considered. When compared to a conventional intake, the flow in this configuration is much more susceptible to separation and insufficient straightening within the intake. Hence a stronger interaction between the fan and the intake is expected. The key objective of the current paper is to explore the two-way interactions between the short intake and fan in detail. This includes the effect of fan on intake AoA tolerance (FoI) and the effect of flow distortion induced by the intake on the fan-performance (IoF). This is in contrast to the earlier studies, where one-way interaction of FoI has received considerable attention on conventional intakes. In addition to the stagnation pressure distortion explored in the context of BLI engines, in the current work, the fan also experiences a strong static pressure distortion under high AoA. Interaction between the intake and fan also depends on the spectral gap between the shock unsteadiness on intake lip and the rotor passing frequency. Hence, this aspect is also investigated in the current study.

The paper is organized as follows. Firstly, the numerical setup is discussed providing the details of the test case, solver and simulation parameters. It is followed by the discussion on the isolated performance of the fan-alone and intake-alone configurations. Validation of the numerical results with the measurements is presented in this section. Subsequent sections present a detailed analyses of the two-way interactions. Section on FoI explores the effect of fan on AoA tolerance and the spectral gap between various characteristic frequencies. On the other hand, section on IoF delves into the fan power and sector analysis as the rotor sweeps through the distortion and the mechanisms by which the inlet distortion affects fan performance. Summary and conclusions are provided in section 6 .

\section{Numerical Setup \\ Test case and boundary conditions}

Figure 1(b) shows the schematic of the test case considered for the current study. An intake of diameter $D$ is placed in a cylindrical domain of dimensions $D_{d} \times L_{d}=9 D \times 7 D$; where $D_{d}$ and $L_{d}$ are the diameter and length of the computational domain respectively. Previous studies on the intakefan interactions $[12,14]$ considered a conventional intake with $L / D=0.5$, where $L$ is the distance from highlight to the fan-face and $D$ is the diameter of the intake. Unlike these studies on a conventional intake, the current study considers a relatively shorter intake which is coupled to a modern high bypass civil aeroengine fan. Thus, a significantly stronger interaction between the intake and fan is expected than the unsteady interaction reported in Cao et al. [12]. The values of $L / D$ and the fan-pressure ratio for the current configuration are not disclosed for proprietary reasons. Also, the short intake considered in this study is a real design unlike the 'model' short intakes studied in [12] where the diffusion component of the intake has been purposefully ignored. Due to circumferential averaging involved in the low-fidelity body force approaches to model smeared geometries [12,23], the events within blade passages cannot be captured. The current study overcomes this limitation by focusing on the high fidelity unsteady RANS simulations at varying AoA's to capture the effects of the inlet on fan and vice versa.

Following boundary conditions (BCs) are imposed on the domain boundaries: a) no-slip on the intake, spinner walls and fan blades b) Mach number, incidence angle and atmospheric conditions of density, pressure and temperature (at an altitude of $\approx 16000 \mathrm{ft}$ ) in the far-field and c) mass flow condition with/without radial equilibrium at the exit boundary for the intake-only/intake-fan cases respectively.

\section{Solver}

Rolls-Royce's in-house solver HYDRA, which is a second order unstructured finite volume code, is used to carry out all the simulations reported in this paper. The solver is suitable to carry out multi-fidelity simulations including Reynolds-averaged Navier-Stokes (RANS)/ unsteady RANS/ Large Eddy Simulations (LES) and has been extensively validated over a wide range of test cases and complex flows $[24,25]$. The turbulent eddy viscosity in the highly strained regions of the flow is estimated using the standard Spalart-Allmaras (SA) turbulence model. Low Mach number preconditioning is also used to improve the predictions in the low-Mach separated zones.

\section{Meshing and simulation parameters}

The near-wall regions on the intake, spinner and the fanblade passages are resolved using the structured hexahedral elements while the free-stream domain is resolved using the tetrahedral elements. This hybrid meshing strategy is much more economical when compared to a fully structured mesh. It minimizes the grid requirement in the free-stream (by $\approx$ $30 \%$ ) and accurately resolves the critical flow features on the intake lip (normal shock and the separated shear layer). 
The total mesh size for the full annulus unsteady RANS calculation (comprising of 18 fan blades and an intake) is around 120 million. Each blade passage has been resolved spatially with 6 million cells and temporally using 60 physical time steps (= 1080 time steps per revolution). A grid sensitivity study has been carried out to arrive at these values. Three different levels of mesh sizes, 3M, 6M and 12M, have been used to study the mesh independence. RANS calculations on the single blade passage have been carried out. HYDRA with standard SA turbulence model was used. Figure 3(a) and 3(b) shows the radial variation of the axial velocity (non-dimensionalized with the tip speed of the blade, $U_{\text {tip }}$ ) at the rotor inlet and exit. Predictions from the three different meshes are compared. It can be seen that, the results given by $6 \mathrm{M}$ and $12 \mathrm{M}$ mesh show little difference on the flow distribution. It thus can be concluded that $6 \mathrm{M}$ mesh per blade passage is sufficient for the low speed fan RANS calculations.

For every test case, the simulation is run for 9 full revolutions followed by time-averaging the solution in the final revolution. To ensure convergence of the solution, power variation has been monitored on a single fan-blade for the last three revolutions (see section 5.1 for further details). Figure 3(c) plots the circumferential variation of the instantaneous power, $\mathbf{P}_{n d}$. Evidently, the variation of power is periodic in time for the $7^{\text {th }}, 8^{\text {th }}$ and $9^{\text {th }}$ revolutions, thus demonstrating the global convergence of the flow-field.

\section{Performance Under Isolation}

\subsection{Rotor-alone configuration}

The performance of the rotor-alone configuration in a uniform, clean flow is estimated using steady simulations. As mentioned in the previous section, a grid size of $6 \times 10^{6}$ elements are used within rotor passage.

The characteristic maps of the total pressure ratio and efficiency are computed at the design speed. Figure 2(a,b) compares the values predicted by CFD against the measurements. The deviation in predictions are within $1.3 \%$ for the total pressure ratio and $1 \%$ for the efficiency. In addition, Fig. 2(c,d) also compares the radial traverses of stagnation pressure and temperature downstream of the rotor at $x \approx 0.5 C_{x}$. The profiles are compared at three critical operating conditions: near stall (A), peak efficiency (B) and near choke (C). Points A, B, C are also marked in Fig. 2(b) for reference. The agreement between CFD and experiments is clearly encouraging. More specifically the sensitivity of the tip flow to the operating condition is consistenly predicted by CFD, thereby validating the numerical framework. Additional simulations are also carried out with an outlet guide vane, OGV (not reported here for brievity). However, its influence on the characteristic map and radial profiles is observed to be minimal.

\subsection{Intake-alone configuration}

As discussed in the introduction, the shock strength on the intake lip increases with increasing angles of attack
(AoA), thereby forcing the flow to separate at the bottom dead centre (BDC). Steady simulations are carried out on an isolated short-intake at different AoAs. The objective is to detect the critical angle at which the flow separates on the lip. A detailed validation of this CFD approach (Isentropic Mach number distributions and distortion levels at the fanface with increasing AoA) against the measurements on a conventional intake can be found in Cao et. al. [12]. Total pressure distortion at the fan-face is quantified using a coefficient, DC60, given as:

$$
D C 60=\frac{P_{0}-P_{0,60}}{P_{0}-P}
$$

Here, $P_{0}$ and $P$ are the area-weighted average total and static pressures at the fan face. A $60^{\circ}$ sector with highest level of distortion (i.e. least value of mean total pressure) is identified at the fan-face. $P_{0,60}$ in Eq. 1 corresponds to the mean total pressure within this sector. DC60 measures the difference between average $P_{0}$ at the intake inlet and the worst $60^{\circ}$ at the aerodynamic interface plane. The flow is considered to be separated when the DC60 exceeds a critical threshold $D C 60_{c r}$. For the intake-alone configuration, Fig. 4(a) plots the variation of non-dimensional distortion coefficient (given by $D C 60_{n d}=D C 60 / D C 60_{c r}$ ) with increasing AoA. Note that, in the figure, abscissa shows the deviation in AoA, $\Delta \alpha_{r e f}=\alpha_{i}-\alpha$; where $\alpha_{i}$ is the absolute value of AoA and $\alpha$ is the angle at which flow has separated. A sharp increase in the distortion coefficient is evident once the flow over the intake lip undergoes shock-induced separation beyond critical AoA. This observation of a steep increase in the distortion is consistent with the previous studies on conventional intakes $[12,26]$. When compared to a short-intake, conventional intakes separate at a relatively higher AoA due to a thicker lip. An inset plot in Fig. 4(a) shows the contours of Mach number at the fan-face for $\alpha+2^{\circ}$. As expected, the flow field is symmetric about the $z=0$ plane. The flow approaching the intake at an AoA turns into the axial direction (i.e. along the engine axis) once it enters the intake. Due to the subsequent streamline curvature, flow acceleration is relatively higher in the lower half of the annulus when compared to the upper half.

It is worth noting that apart from DC60, a variety of distortion indices exist in literature. Campbell [27] compared several such indices and found Aerospace Recommended Practice (ARP)-1420 to provide accurate correlations. In addition to the intensity of pressure distortion, this descriptor accounts for the shape of the distortion pattern. For a given circumferential ring number $i$ (see [20]), the circumferential distortion intensity $\left(\Delta P_{c} / P\right)_{i}$ and radial distortion intensity $\left(\Delta P_{r} / P\right)_{i}$ are given by: 


$$
\begin{array}{r}
\left(\Delta P_{c} / P\right)_{i}=\frac{\left(P_{\text {avg }-i}-P_{\text {avglow-i }}\right)}{P_{\text {avg }-i}} \\
\left(\Delta P_{r} / P\right)_{i}=\frac{\left(P_{\text {face }- \text { avg }}-P_{\text {avg }-i}\right)}{P_{\text {face }- \text { avg }}} \\
P_{\text {avg }-i}=(1 / 360) \int_{0}^{360} P(\theta) d \theta \\
P_{\text {avg-low }-i}=\left(1 /\left(\theta_{2}-\theta_{1}\right)\right) \int_{\theta_{1}}^{\theta_{2}} P(\theta) d \theta
\end{array}
$$

Here, $\theta_{1}$ and $\theta_{2}$ are the angles between which the total pressure is lower than $P_{\text {avg }-i}$ and $P_{\text {face-avg }}$ is the average pressure on the fan-face at which distortion is estimated. Figure 4(b) shows the variation of both the circumferential and radial distortion intensities for the intake-alone configuration at $\alpha+2^{\circ}$.

\section{Effect of Fan on Intake Distortion (FoI)}

Simulations are carried out on the coupled configuration for increasing AoA over the intake. Mixing plane approach is initially used to obtain a representative flow field at a lower AoA. At this AoA, the flow remains attached over the intake lip. The solution is subsequently used to initialize the unsteady RANS simulation.

Figure 4(a) compares the predictions of the distortion coefficient, $D C 60$, for the powered intake against an isolated intake configuration. An inset plot in Fig. 4(a) shows the contours of Mach number at the fan-face for $\alpha+6^{\circ}$. It is evident that the presence of a fan has effectively suppressed the distortion. AoA tolerance of the powered-intake configuration has increased by $\approx 4^{\circ}$ when compared to an isolated intake. This observation of increase in the separationfree AoA tolerance of a powered intake is consistent with both the experimental and numerical studies in the literature $[3,11-13,28]$. As noted by Cao et. al. [12], the rotor redistributes the mass-flow upstream of the fan-face (see Fig. $3(\mathrm{a})$, accelerating more flow at the casing relative to the hub. This additional acceleration alleviates distortion. It is also apparent from Fig. 4(a) that once the flow separates over the intake lip, the distortion at the fan-face grows gradually for a powered intake. This is in contrast to a steep increase in the distortion noted for an isolated-intake. Moreover, the flow field is asymmetric about the $z=0$ plane due to the rotor induced mass-flow redistribution. Reduction in the fan-face distortion due to rotor is also apparent from Fig. 4(b). Both the circumferential and radial distortion intensities in terms of ARP-1420 for $\alpha+6^{\circ}$ are lower than the corresponding values of the intake-alone configuration at $\alpha+2^{\circ}$.

It should be noted that $D C 60$ in Fig. 4 is estimated based on the time-averaged flow field at the fan-face. However, the phenomenon of shock-boundary layer interaction is inherently unsteady, specifically at larger AoA's when the shock is strong enough to promote separation. Significant research has been devoted in the literature to address and mitigate the low-frequency self-sustained shock oscillations (also referred as transonic buffet, see $[29,30])$. The surface pressure fluctuations can vary between $\pm 20 \%$ of the mean value leading to detrimental effects of lift reduction and increased structural loads. However, Coschignano and Babinsky [9] note that most of these studies are confined to the flows over aerofoils/wings while very few explored the shock formation over engine intake lip [31,32]. In particular, it is crucial to (a) quantify the pressure fluctuations as it has serious implications on the rotor's structural integrity and (b) determine the extent of the spectral gap between the frequency of the shock unsteadiness and the blade-passing frequency of the rotor. If the natural frequency of the shock unsteadiness is a sub or super-harmonic of the blade passing frequency, it can lead to resonance and hence further damage to the rotor blades. To illustrate this, Fig. 5 presents the contours of Mach number on the selected planes for the case with $\mathrm{AoA}=\alpha+6^{\circ}$. Shock induced separation can be visualised on $y=0$ plane. The subsequent circumferential distortion transferred to the fan can be monitored on the fan-face plane.

Data is recorded on both these planes for three full revolutions of the rotor. This data is represented in the form of space-time plots (ST plots) along the lines labelled in Fig. 5. Figure 6(a) shows contours of Mach number along the intake-lip on $y=0$ plane and Fig. 6(b) shows the contours of stagnation pressure at the fan-face, along a $120^{\circ}$ sector at the BDC. $S_{n d}$ in Fig. 6(a) represents the distance along the intake, non-dimensionalized by the lip-length, $L_{\text {lip }}$. ST plots are particularly useful to illustrate the propagation of different disturbances in space and time. Both high and low-frequency variations of the monitored quantities are evident in the ST plots (marked accordingly in the figures). Of these, the low-frequency variation is a consequence of the shock oscillation over the intake lip. Corresponding variation in the circumferential and radial extent of the distortion at the fan-face is also evident from Fig. 6(b,c). Conversely, high frequencies occur due to the blade passing, particularly when the bow-shock at the leading edge of the rotor sweeps through the distortion. Hence, the DC60 at the fanface varies with time unlike a fixed mean value shown in Fig. 4. The typical variation due to the low-frequency unsteadiness is estimated to be around $\pm 5-8 \%$ of the time-averaged value.

Figure 7 plots the Power spectral density (PSD) of pressure fluctuations to describe how the mean squared value of pressure is distributed in frequency. Pressure signals are recorded both pre-shock (probe P1) and post-shock (probe P2) locations, marked in Fig. 5. PSD is scaled using the reference dynamic pressure in the free-stream, $\gamma M_{r e f}^{2} P_{r e f}$, while the frequency is normalized with the number of full rotor revolutions per second, $f_{r}$. Distinct high and low frequencies peaks are captured by the probe $\mathrm{P} 2$. Of these, low frequency peak at $f / f_{r} \approx 1.4$ corresponds to the shock unsteadiness and the high frequency peak at $f / f_{r} \approx 18$ indicates the blade passing. Note that, given the scaling with $f_{r}$, this value of 18 corresponds to the number of rotor blades. From this discussion, it is clear that a sufficient 'spectralgap' exists between the shock unsteadiness and blade pass- 
ing, with almost an order-of-magnitude difference in the corresponding frequencies. Hence the possibility of resonance due to the superharmonic coupling between frequencies of the rotor passing and the unsteadiness of the primary shockinduced shear layer can be ruled out. Infact, from Fig. 6(b,c), it is clear that the bow-shock of the rotor passing induces high frequency unsteadiness causing the DC60 to vary between $\pm 0.4-0.6 \%$ of the local $D C 60$ value. Hence, its effect is secondary when compared to the $5-8 \%$ DC60 variation due to low-frequency shock unsteadiness on the intake lip. This dynamic component of the distortion is crucial while designing the steady screens to simulate the pressure distortion in the inlet wind tunnel tests. As noted by Cousins and Davis [20], such screens should be designed at RMS or peak value of the dynamic measurement instead of designing at the steady state distortion values. Interestingly, no distinct frequencies are observable from the signal at probe P1 corresponding to the pre-shock location. It indicates that the shock filters out the information of the shear-layer unsteadiness and blade passing to propagate to this location.

\section{Effect of Inlet Distortion on Fan Performance (IoF)}

In this section, full-annulus unsteady simulations are further processed to examine the effect of inlet distortion on the fan performance. This includes discussion on the power variation as the fan sweeps through the distortion, sectorwise analysis of the rotor characteristic and the mechanisms through which distortion affects the fan-performance.

\subsection{Fan Power:}

During the simulation, the total force, $F$ (pressure drag + viscous drag), acting on selected fan blades is monitored. Subsequently, torque $(\tau)$ and power $(\mathbf{P})$ are estimated using:

$$
\tau=\int r \times F . d A \quad \mathbf{P}=\tau \Omega
$$

$r, d A$ and $\Omega$ in the above equation represent the local blade radius, elemental surface area and angular velocity of the blade rotation respectively. For increasing AoA onto the intake, Fig. 8 plots the circumferential variation of the nondimensional power $\left(\mathbf{P}_{n d}\right)$ monitored on a fan blade as it rotates around the annulus. Mean power at $\alpha+3^{\circ}$ is used as a reference to normalize the instantaneous power. Key observations that can be made from this figure are: a) 'large scale' sinusoidal variation (once per revolution) of the power about the mean value, with the amplitudes reaching as high as $\pm 10 \%$ and $b$ ) 'small scale' peaks/troughs within a localized sector between $\theta \approx 160-230^{\circ}$, for the AoA's of $\alpha+4^{\circ}$ and $\alpha+6^{\circ}$. Since the intake operates at an AoA, the flow at the fan-face is largely non-uniform (refer to the inset plots in Fig. 4). The resultant non-uniformity in the flow coefficient and incidence, affects the local operating condition of the fan-blade. This inviscid (or potential field) effect contributes to the 'large scale' variation in the power signal which occurs once every revolution. On the other hand, as discussed in section 4, flow over the intake lip experiences a strong shock-induced separation at larger AoA. For AoA's of $\alpha+4^{\circ}$ and $\alpha+6^{\circ}$, the distortion shows a relative increase. Significant loss in the stagnation pressure occurs at the bottom dead centre of the intake. When the fan-blade of interest sweeps through this distorted sector, local spikes/troughs appear in the power signal. Hence, the viscous effect contributes to the 'small scale' local power variation. To summarize, both the inviscid and viscous effects enhance the flow non-uniformity at the fan-face thereby affecting the performance of the fan. As expected, the mean fan-performance has deteriorated at higher AoA's.

\subsection{Sector Analysis}

In this section, a sector-wise performance analysis of the rotor has been carried out. This approach is useful to examine the rotor performance as it sweeps through the distortion. In addition to the stagnation pressure distortion addressed in earlier studies $[15,16]$, rotor in the current study also encounters a static pressure distortion, since the engine operates under high-incidence (see inset plots in Fig. 4). Following Fidalgo et al. [15] and Gunn et al. [16], a time-averaged flow is firstly obtained in the absolute frame of reference. This is a non-trivial exercise and requires a special treatment of the rotor domain. It involves (a) saving the unsteady snapshots of the rotor domain for one full revolution (b) interpolating each of these solutions on to a stationary grid in the absolute frame of reference and (c) time averaging the solution variable, $v$, on the stationary grid as $\bar{v} \approx(1 / N) \sum_{1}^{N} H(t) v(t)$. Here, $\bar{v}$ is the time-averaged variable, $N$ is the total number of time steps and $H(t)$ is the gate function which is set to zero within the blade and one elsewhere. This time-averaging approach based on gate function, developed by Adamczyk [33], eliminates the blades in the final solution. The solution is then used to construct the 'mean' streamtubes in the rotor domain. Figure 9 shows the inlet and exit planes extracted upstream and downstream of the rotor. Four rakes on the inlet plane divide the full annulus into four equal $90^{\circ}$ sectors. These are referred to as 'inlet sectors'. From each of these rakes, streamlines are released and tracked through the rotor domain. The locus of points where the streamlines intersect with exit plane, and corresponding shape of the 'exit sectors', are then computed. Following [15], mass flow rates are measured through the inlet sector and the corresponding exit sector. The mass flow difference between these sectors is found to be within $\pm 0.2 \%-1 \%$, thereby confirming the accuracy of this streamtube extraction approach. Similar activity has been carried out at different AoA's. It is interesting to note from Fig. 9 that AoA had little influence on the shape of the exit sectors, except that the size of sector $I V$ is marginally larger at higher AoA of $\alpha+6^{\circ}$.

Figure 10(a) plots the rotor performance on the characteristic map (i.e. pressure ratio vs mass-flow) as it sweeps through each of these four sectors for $\mathrm{AoA}=\alpha$. Each of the operating points in sectors I-IV is shown using square symbols. The steady characteristic for the clean flow at design speed and zero AoA is also overlaid in the figure for compar- 
ison. Locus of the local operating points encircle an 'orbit'. Evidently, the local operating condition of the rotor varies considerably within the orbit, even if the flow over the intake lip remains attached at $\mathrm{AoA}=\alpha$. This is attributed to the fact that the flow approaching the intake at AoA results in a circumferentially non-uniform distribution of pressure, flow coefficient and blade incidence on the inlet plane. It is interesting to note that, despite such local variations, the mean operating point still lies on the steady characteristic map. On the other hand, Fig. 10(b) illustrates the change in orbit shape with a gradual increase in AoA onto the intake. In the figure, symbols with cross indicate the corresponding mean operating point. When compared to the case with $A o A=\alpha$, the mean pressure ratio decreased for the AoA's of $\alpha+3^{\circ}$, $\alpha+4^{\circ}$ and $\alpha+6^{\circ}$. At $\alpha+6^{\circ}$ condition, significant losses are incurred from the shock-induced separation (a) on the intake lip and (b) within the blade passages (due to passage shocks). This has resulted in a large decrease in the pressure ratio for $\mathrm{AoA}=\alpha+6^{\circ}$. For this condition, the mass flow has also reduced in sectors I, IV due to distortion which gets redistributed to sectors II, III. Note that the resolution of the operating 'orbits' can be increased further by tracking more than four inlet and exit sectors. This is illustrated in Figure 10(a), where each of the sectors I-IV is further sub-divided into four sub-sectors. The operating condition of these subsectors is shown using circular symbols around its local mean operating point (indicated using square symbols). The shape of the highly resolved orbit is almost elliptical instead of the quadrilateral orbit of the sectors I-IV. However, as will be shown in the subsequent section, the loss mechanisms can be addressed in enough detail using four sectors and hence we restrict the number of sectors to four.

\subsection{Mechanisms}

To provide further insights into the physical understanding of the fan performance in each of the four sectors (I-IV), Fig. 11 presents the contours of (i) flow coefficient deviation, $\Delta \Phi$, in frames (a,c) and (ii) incidence angle, $\beta_{i n}^{\circ}$ in frames $(\mathrm{b}, \mathrm{d})$. The deviation in flow coefficient is defined as $\Delta \Phi=\Phi(r, \theta)-\Phi_{a v g}(r)$, where $\Phi_{a v g}(r)$ is the circumferential averaged flow coefficent at a given radius. Two AoA's corresponding to attached and separated flow (AoA $\left.=\alpha+6^{\circ}\right)$ over the intake lip are shown. These frames are extracted upstream of the rotor. For clarity, inlet sectors are also marked in the figure with solid lines which are consistent with those shown in Fig. 9. From the contour plots, a large scale variation of $\Delta \Phi$ and $\beta_{i n}^{\circ}$ is clearly evident in the circumferential and radial directions across all the four sectors at both AoA's. Sinusoidal variations of the fan power in Fig. 8 and the 'orbits' in fan characteristic map in Fig. 10 are a consequence of these variations in $\Delta \Phi$ and $\beta_{i n}^{\circ}$. Two mechanisms are identified for such large scale flow variations on the inlet plane, which include (a) variation in the flow coefficient, $\Phi$, due to streamline curvature/intake loading and (b) variation in the flow incidence onto the fan blade due to up-wash effect of incoming flow.
Streamline curvature effect: The streamlines approaching the intake at high AoA turn to axial direction along the engine axis. This streamline curvature induces a significant pressure gradient (or intake loading) along its local radius of curvature. The flow at the top dead centre (TDC) of the intake experiences high pressure, while it experiences a relatively lower pressure near the bottom dead centre (BDC). The flow coefficient consequently adjusts to this pressure gradient, resulting in a lower $\Phi$ at the TDC (sector II) and higher $\Phi$ at the BDC (sector IV) of the intake. A change in flow coefficient also alters the local blade incidence, $\beta_{i n}^{\circ}$, to higher values in sector II and lower values in sector IV. This combined variation of both $\Delta \Phi$ and $\beta_{i n}^{\circ}$ explains the local operating condition of the fan in Fig. 10 i.e., low flow rate with high pressure ratio in sector II and high flow rate with low pressure ratio in sector IV.

Upwash effect: For a short-intake configuration, the incoming flow fails to entirely turn into the axial direction. Subsequently, the fan encounters a residual upwash flow angle which contributes to the incidence distortion. Infact Peters et al. [3] identified such a reduction in the flow straightening through the inlet as one of the limiting factors for the short inlet design. Since the fan rotates in the clockwise direction (when viewed from the inlet), it can be shown from the velocity triangles that the up-wash flow results in a lower flow incidence to the fan blade in sector I and a higher incidence in sector III. This effectively imposes a co-swirl and counterswirl to the fan in zones I and III respectively. Hence, as noted in Fig. 10, the fan characteristic shifts towards a lower mass flow and pressure ratio in sector I and a higher mass flow with higher pressure ratio in sector III. With further increase in AoA to $\alpha+6^{\circ}$, Fig. 11 (c) and (d) shows similar patterns of $\Delta \Phi$ and $\beta_{i n}^{\circ}$. The variation is however much more stronger than $\mathrm{AoA}=\alpha$. Furthermore, shock induced separation on the bottom lip of the intake lip is noticeable for AoA $=\alpha+6^{\circ}$ case. This results in a local reduction of the flow coefficient and increase in the flow incidence to the blade. Figure 12(a,b) illustrates the above described mechanisms of streamline curvature and upwash effect by means of a sketch. Both these mechanisms are at play in sectors I-IV at the fanface thereby affecting the fan performance.

Wake thickening: Both the shock induced flow separation on the intake lip and the consequent change in the flow incidence has a detrimental effect on the performance of the fan blade as it sweeps through the distortion. Figure 13 illustrates this by comparing the relative Mach number contours downstream of the fan at AoA's of $\alpha$ and $\alpha+6^{\circ}$. Exit sectors from Fig. 9 are also overlaid for clarity. This figure highlights the key features of the instantaneous wakes shed from each of the fan blades. It can be observed that considerably thicker wakes are shed at higher AoA when compared those at lower AoA. The wakes are thicker across the entire span of the fan blades, specifically in sectors IV and I.

The underlying flow physics resulting in such a wake thickening varies from mid-span to the blade tip. Figure 14 shows contours of relative Mach number at the mid-span of the blades. For a given radius, both the circumferential and axial variation of the flow properties can be conveniently 
shown in these unwrapped plots. Note that the axial coordinate is non-dimensionalized with the tip blade chord. It is evident that the fan blades in $\operatorname{sector} \operatorname{IV}\left(\theta^{\circ} \approx 150-220^{\circ}\right)$ show a strong passage shock particularly at AoA $=\alpha+6^{\circ}$. The blades in this sector choke around the mid-span due to an increase of the flow coefficient. Two factors contribute to this increase of $\Phi$ at the mid-span which include: (a) increase in $\Delta \Phi$ within sector IV due to a relatively stronger streamline curvature effect (or intake loading effect) at higher AoA and (b) shock induced flow separation on the BDC of the intake lip which drives more flow to pass through the midspan than the casing. The shock waves generated in the passage trigger boundary layer separation on the suction side of the blade, hence resulting in thicker wakes at the mid-span.

On the other hand, thickening of the wake closer to the blade tip is attributed to the distortion transfer. Figure 15 shows the contours of relative Mach number extracted at $90 \%$ span. Low Mach number region notable between $160-200^{\circ}$ is essentially due to the shock-induced separation on the BDC of the intake. Flow separation causes a local drop in both the flow coefficient and the relative Mach number. As the distorted flow convects through the blade passages, it experiences a tangential force in the direction of rotation of the blade rows. The distortion convects out of the blade passage and propagates downstream as indicted by the dashed line in the figure. This is similar to the mixed distortion transfer observed by Fidalgo et al. [15] and Gunn et al. [34] in the context of boundary layer ingesting fans under zero AoA. It is also worth mentioning that the response of the rotor to varying inlet condition is not instantaneous. As noted by Cousins [35], it is a function of the time for which the blade passes through the distortion and the time taken by the fluid particle to reach the throat in the blade passage. In the current simulations, since fluid particles reach the throat faster than the time the rotor takes to sweep through the distortion (particularly for the case $\alpha+6^{\circ}$ ), the diffusion process in the rest of the blade passage is disrupted. In such cases the flow separates in the passage as evident from Figure 15. This limits the pressure rise capability and the blade response is maximized.

To summarize, both distortion transfer near the blade tip and the at the choking of the blade passages in the mid-span contribute to the overall thickening of the wake shed from the fan-blades in sectors IV and I. This results in a substantial drop in the pressure ratio as noted in Fig. 10. Although, shock induced separation on intake lip influences fan performance, the current study clearly demonstrates (and also reconfirms the findings of Peters et al. [3]) that insufficient flow straightening could be one of the limiting factors for the short-intake design strategy, specifically at higher angles of attack. It should be noted that the upstream potential field effect of the pylon and Fan exit guide vanes (FEGVs) are not considered in this study and are beyond the scope of the current work. Peters [36] alleviates the upstream influence of pylon using non-axisymmetric FEGVs. Additional investigations in these lines with wing, pylon and splitter will be helpful to further explore the installation effects.

\section{Summary and Conclusions}

In this paper, we present a numerical study on the interaction between a fan with the flow over an intake under high incidence. We consider a new short intake design coupled to a modern fan in a high-bypass ratio civil engine. The interaction is much significant than that on a conventional intake (with larger $L / D$ ). The performance of both the intake-alone and rotor-alone configurations are examined under isolation. These include: a) estimating the AoA at which the flow separates on an intake lip and b) favourably validating the fan characteristic and radial profiles against the measurements. Subsequently, two-way interactions between intake and fan are discussed in detail. This includes the effect of fan on intake AoA tolerance (FoI) and the effect of flow distortion induced by the intake on the fan-performance (IoF).

In the FoI scenario, the rotor effectively redistributes the mass flow at the fan-face. The AoA tolerance of the shortintake design has increased by $\approx 4^{\circ}$ when compared to the intake-alone configuration; which is consistent with the previous studies in the literature. Low-frequency shock oscillation on the intake lip and high-frequency rotor passing has been captured using the space-time (ST) plots and power spectral density (PSD) of pressure fluctuations. The results show a sufficient spectral gap between the shock unsteadiness and blade passing, with almost an order of magnitude difference in the corresponding frequencies.

In the IoF scenario, both the 'large' (once per revolution) and 'small' scale distortion (in a distorted sector) induced by the intake results in a non-uniform inflow to the rotor. Sector analysis reveals a substantial variation in the local operating condition of the fan as opposed to its steady characteristic. Streamline curvature, upwash and wake thickening are identified to be the three key factors affecting the fan performance.

'Large-scale' non-uniformity (once per revolution) is an inviscid effect which results from the AoA at which the intake operates. This inviscid effect is a consequence of the circumferential and radial variation of both the flow coefficient, $\Phi$ and the flow incidence, $\beta$ to the fan blade. While $\Phi$ changes due to the streamline curvature/intake loading, $\beta$ changes due to the upwash of the incoming flow.

'Small-scale' distortion $\left(O\left(10-60^{\circ}\right)\right)$ occurs specifically at higher AoA's. This is a viscous effect resulting from the shock-induced separation on the intake lip. Subsequent distortion transfer at the casing and strong passage shocks in the mid-span contribute to the overall thickening of the fan wakes and a substantial drop in the pressure ratio.

In addition to the shock induced separation on the intake lip, the current study shows that shorter intakes are much more prone to the upwash effect at higher AoA. Insufficient flow straightening along the engine axis is reconfirmed be one of the limiting factors for the short-intake design.

\section{Acknowledgements}

This project was funded by Innovate UK and RollsRoyce. The authors wish to acknowledge the computational time granted on ARCHER (UK Supercomputing facility) via 
the EPSRC RAP call of spring 2017. Dr. Nagabhushana Rao Vadlamani gratefully acknowledges the Bowring research fellowship from the St. Catharine's college, Cambridge.

$\begin{array}{ll}\text { Nomenclature } \\ D & \text { Diameter of the intake } \\ D_{d} & \text { Diameter of computational domain } \\ D C 60 & \text { Distortion coefficient } \\ f & \text { frequency } \\ f_{r} & \text { fan rotational speed } \\ H(t) & \text { gate function } \\ L_{d} & \text { Length of computational domain } \\ M_{r e f} & \text { Reference Mach number } \\ P_{r e f} & \text { Reference freestream pressure } \\ \mathbf{P}_{n d} & \text { Instantaneous non-dimensional power } \\ P_{0} & \text { Area-weighted total pressure } \\ P_{0,60} & P_{0} \text { in } 60^{\circ} \text { distorted sector } \\ P & \text { Area-weighted static pressure } \\ L_{l i p} & \text { Length of intake lip } \\ \text { Greek } & \text { Symbols } \\ \Phi & \text { Flow coefficient } \\ \alpha & \text { Angle of attack } \\ \beta_{i n} \quad \text { Incidence angle } \\ S_{n d} \quad \text { Distance along intake lip } \\ \tau \quad \text { Torque } \\ \theta \quad \text { Circumferential coordinate } \\ \text { Acronyms } \\ \text { AoA } & \text { Angle of attack } \\ \text { BPR } & \text { Bypass ratio } \\ \text { BDC } & \text { Bottom dead centre } \\ \text { CFD } & \text { Computational fluid dynamics } \\ \text { FPR } & \text { Fan pressure ratio } \\ \text { OGV } & \text { Outlet guide vane } \\ \text { RANS } & \text { Reynolds Averaged Navier-Stokes } \\ \text { TDC } & \text { Top dead centre } \\ \text { LES } & \text { Large Eddy Simulations } \\ \text { SA } & \text { Spalart Allmaras } \\ \text { ST } & \text { Space Time plot } \\ \text { SWBLI } & \text { Shock wave boundary layer interaction }\end{array}$

SWBLI Shock wave boundary layer interaction

\section{References}

[1] Hall, C. A., and Crichton, D., 2007. "Engine design studies for a silent aircraft". Journal of Turbomachinery, 129(3), pp. 479-487.

[2] Cumpsty, N. A., 2010. "Preparing for the future: reducing gas turbine environmental impact - igti scholar lecture". Journal of turbomachinery, 132(4), p. 041017.

[3] Peters, A., Spakovszky, Z. S., Lord, W. K., and Rose, B., 2015. "Ultrashort nacelles for low fan pressure ratio propulsors". Journal of Turbomachinery, 137(2), p. 021001.

[4] Thollet, W., Dufour, G., Carbonneau, X., and Blanc, F., 2016. "Body-force modeling for aerodynamic analysis of air intake-fan interactions". International Journal of Numerical Methods for Heat \& Fluid Flow, 26(7), pp. 2048-2065.

[5] Lesser, A., and Niehuis, R., 2014. "Transonic axial compressors with total pressure inlet flow field distortions". In Proceedings of ASME Turbo Expo, June 1620, 2014, Dusseldorf, Germany, pp. V01AT01A036V01AT01A036.

[6] Davis, M. W., and Cousins, W. T., 2011. "Evaluating complex inlet distortion with a parallel compressor model: Part 2-applications to complex patterns". In Proceedings of ASME Turbo Expo, June 6-10, 2011, Vancouver, Canada, pp. 13-23.

[7] Freeman, C., and Rowe, A. L., 1999. "Intake engine interactions of a modern large turbofan engine". In ASME 1999 International Gas Turbine and Aeroengine Congress and Exhibition, June 7-9, 1999, Indianapolis, USA, pp. V001T01A007-V001T01A007.

[8] Makuni, T. E., Babinsky, H., Slaby, M., and Sheaf, C. T., 2015. "Shock Wave-Boundary-Layer Interactions in Subsonic Intakes at High Incidence". In 53rd AIAA Aerospace Sciences Meeting, 5-9 January, Kissimmee, Florida, American Institute of Aeronautics and Astronautics.

[9] Coschignano, A., Babinsky, H., Sheaf, C., and Zamboni, G., 2019. "Normal-shock/boundary-layer interactions in transonic intakes at high incidence". AIAA Journal, pp. 1-14.

[10] Hodder, B., 1981. "An investigation of engine influence on inlet performance. [conducted in the ames 40-by 80foot wind tunnel]". NASA CR-166136.

[11] Larkin, M. J., and Schweiger, P. S., 1992. "Ultra high bypass nacelle aerodynamics inlet flow-through high angle of attack distortion test". NASA Contractor Report CR - 189149.

[12] Cao, T., Vadlamani, N. R., Tucker, P. G., Smith, A. R., Slaby, M., and Sheaf, C. T., 2017. "Fan-intake interaction under high incidence". Journal of Engineering for Gas Turbines and Power, 139(4), p. 041204.

[13] Carnevale, M., Wang, F., and di Mare, L., 2017. "Low frequency distortion in civil aero-engine intake". Journal of Engineering for Gas Turbines and Power, 139(4), p. 041203.

[14] Carnevale, M., Wang, F., Green, J., and Di Mare, L., 2015. "Lip stall suppression in powered intakes". Journal of Propulsion and Power.

[15] Fidalgo, V. J., Hall, C., and Colin, Y., 2012. "A study of fan-distortion interaction within the nasa rotor 67 transonic stage". Journal of Turbomachinery, 134(5), p. 051011.

[16] Gunn, E. J., Tooze, S. E., Hall, C. A., and Colin, Y., 2013. "An experimental study of loss sources in a fan operating with continuous inlet stagnation pressure distortion". Journal of Turbomachinery, 135(5), p. 051002.

[17] Ochs, S. S., Tillman, G., Joo, J., and Voytovych, D. M., 2016. "Computational fluid dynamics-based analysis of boundary layer ingesting propulsion". Journal of Propulsion and Power, 33(2), pp. 522-530.

[18] Cousins, W. T., Voytovych, D., Tillman, G., and Gray, E., 2017. "Design of a distortion-tolerant fan for a boundary-layer ingesting embedded engine applica- 
tion". In 53rd AIAA/SAE/ASEE Joint Propulsion Conference, 10-12 July, Atlanta, GA, p. 5042.

[19] Frohnapfel, D. J., Ferrar, A. M., Bailey, J., O’Brien, W. F., and Lowe, K. T., 2016. "Measurements of fan response to inlet total pressure and swirl distortions produced by boundary layer ingesting aircraft configurations". In 54th AIAA Aerospace Sciences Meeting, 4-8 January 2016, San Diego, California, USA, p. 0533.

[20] Cousins, W. T., and Davis, M. W., 2011. "Evaluating complex inlet distortion with a parallel compressor model: Part 1 -concepts, theory, extensions, and limitations". In Proceedings of ASME Turbo Expo, June 6-10, 2011, Vancouver, Canada, pp. 1-12.

[21] Ma, Y., Cui, J., Vadlamani, N. R., and Tucker, P., 2018. "Effect of fan on inlet distortion: Mixed-fidelity approach". AIAA Journal, 56(6), pp. 2350-2360.

[22] Ma, Y., Vadlamani, N. R., Cui, J., and Tucker, P., 2019. "Comparative studies of rans versus large eddy simulation for fan-intake interaction". Journal of Fluids Engineering, 141(3), p. 031106.

[23] Watson, R., Cui, J., Ma, Y., Tyacke, J., Vadlamani, N. R., Alam, M. F., Dai, Y., Tucker, P. G., Cao, T., and Hield, P., 2017. "Improved hierarchical modelling for aerodynamically coupled systems". In Proceedings of ASME Turbo Expo 2017, June 26-30, Charlotte, NC USA, pp. V02BT41A056-V02BT41A056.

[24] Lapworth, L., 2004. "Hydra-CFD: A framework for collaborative CFD development". In International Conference on Scientific and Engineering Computation (IC-SEC), July 5-8, Singapore, Vol. 30.

[25] Watson, R., 2013. "Large eddy simulation of cutback trailing edges for film cooling turbine blades". PhD thesis, University of Cambridge, Cambridge, UK.

[26] Carnevale, M., Wang, F., Parry, A. B., Green, J. S., and di Mare, L., 2017. "Fan similarity model for the fanintake interaction problem". In Proceedings of ASME Turbo Expo, June 26-30, 2017, Charlotte, NC USA, pp. V001T01A019-V001T01A019.

[27] Campbell, A. F., 1981. "An investigation of distortion indices for prediction of stalling behavior in aircraft gas turbine engines". PhD thesis, Virginia Polytechnic Institute and State University.

[28] Boldman, D. R., Iek, C., Hwang, D., Larkin, M., and Schweiger, P., 1993. "Effect of a rotating propeller on the separation angle of attack and distortion in ducted propeller inlets". NASA Technical Memorandum 105935.

[29] Lee, B., 2001. "Self-sustained shock oscillations on airfoils at transonic speeds". Progress in Aerospace Sciences, 37(2), pp. 147-196.

[30] Giannelis, N. F., Vio, G. A., and Levinski, O., 2017. "A review of recent developments in the understanding of transonic shock buffet". Progress in Aerospace Sciences, 92, pp. 39-84.

[31] Jakubowski, A., and Luidens, R., 1975. "Internal cowlseparation at high incidence angles". In 13th Aerospace Sciences Meeting, 20-22 January, Pasadena, CA, USA, p. 64.
[32] Chou, D., Luidens, R., and Stockman, N., 1978. "Prediction of boundary-layer flow separation in v/stol engine inlets". Journal of Aircraft, 15(8), pp. 474-481.

[33] Adamczyk, J. J., 1985. "Model equation for simulating flows in multistage turbomachinery". In 30th International Gas Turbine Conference and Exhibit, Houston, pp. 85-GT-226.

[34] Gunn, E., and Hall, C., 2014. "Aerodynamics of boundary layer ingesting fans". In Proceedings of ASME Turbo Expo, June 16-20, 2014, Dusseldorf, Germany, pp. V01AT01A024-V01AT01A024.

[35] Cousins, W. T., 1997. "The dynamics of stall and surge behavior in axial-centrifugal compressors". PhD thesis, Virginia Tech.

[36] Peters, A., 2014. "Ultra-short nacelles for low fan pressure ratio propulsors". PhD thesis, Massachusetts Institute of Technology. 


\section{Figure Caption List}

Figure 1. (a) Key flow features of intake operating under high incidence (b) Computational setup and boundary conditions.

Figure 2. Mesh sensitivity study showing radial traverses of axial velocity non-dimensionalized with the tip speed, (a) upstream and (b) downstream of the rotor. (c) Circumferential variation of power, $\mathbf{P}_{n d}$, on a fan-blade for 3 different revolutions to demonstrate convergence (using $6 \mathrm{M}$ mesh per rotor passage).

Figure 3. Validation of characteristic maps: (a) total pressure ratio (b) efficiency and radial profiles: (c) stagnation pressure (d) stagnation temperature against measurements at selected operating conditions.

Figure 4. (a) Variation of normalized distortion coefficient, $D C 60_{n d}$ with increasing incidence for intake-only and intake-fan configurations. Inset plots show the corresponding contours of Mach number at the fan-face (b) Spanwise variation of ARP-1420 quantifying circumferential and radial distortion.

Figure 5. Instantaneous Mach number distribution on $y=0$ plane and fan-face for $\alpha+6^{\circ}$. Inset plot shows the magnified view of shock-induced separation and subsequent distortion.

Figure 6. Space-Time plot of variation of (a) Mach-number along the intake lip on $y=0$ plane (b) stagnation pressure along the circumference and (c) stagnation pressure along the radial direction for three rotor revolutions.

Figure 7. Power Spectral Density (PSD) of pressure fluctuations measured at probes P1 (pre-shock) and P2 (post-shock).

Figure 8. Circumferential variation of non-dimensional power, $\mathbf{P}_{n d}$, on a fan blade for AoA's: $\alpha+3^{\circ}, \alpha+4^{\circ}, \alpha+6^{\circ}$.

Figure 9. Inlet and exit sectors used to estimate local operating condition of the rotor.

Figure 10. Local operating condition of the rotor, relative to mean point, when flow over intake lip is (a) attached $\left(\right.$ AoA $\left.=\alpha^{\circ}\right)$ and (b) separated $\left(\mathrm{Ao} A=\alpha+6^{\circ}\right)$.

Figure 11. Contours of (a,c) flow coefficient deviation, $\Delta \Phi$ and (b,d) incidence angle, $\beta_{\text {in }}^{\circ}$ for AoA's of $\alpha$ and $\alpha+6^{\circ}$.

Figure 12. Sketch illustrating streamline curvature effect and upwash effect.

Figure 13. Instantaneous wakes shown through the contours of relative Mach number downstream of the fan at AoA's of $\alpha$ and $\alpha+6^{\circ}$. Exit sectors are marked with solid lines for clarity.

Figure 14. Unwrapped blade-to-blade snapshots showing contours of relative Mach number at mid-span for AoA's of $\alpha$ and $\alpha+6^{\circ}$.

Figure 15. Unwrapped blade-to-blade snapshots showing contours of relative Mach number at $90 \%$ span for AoA's of $\alpha$ and $\alpha+6^{\circ}$. 

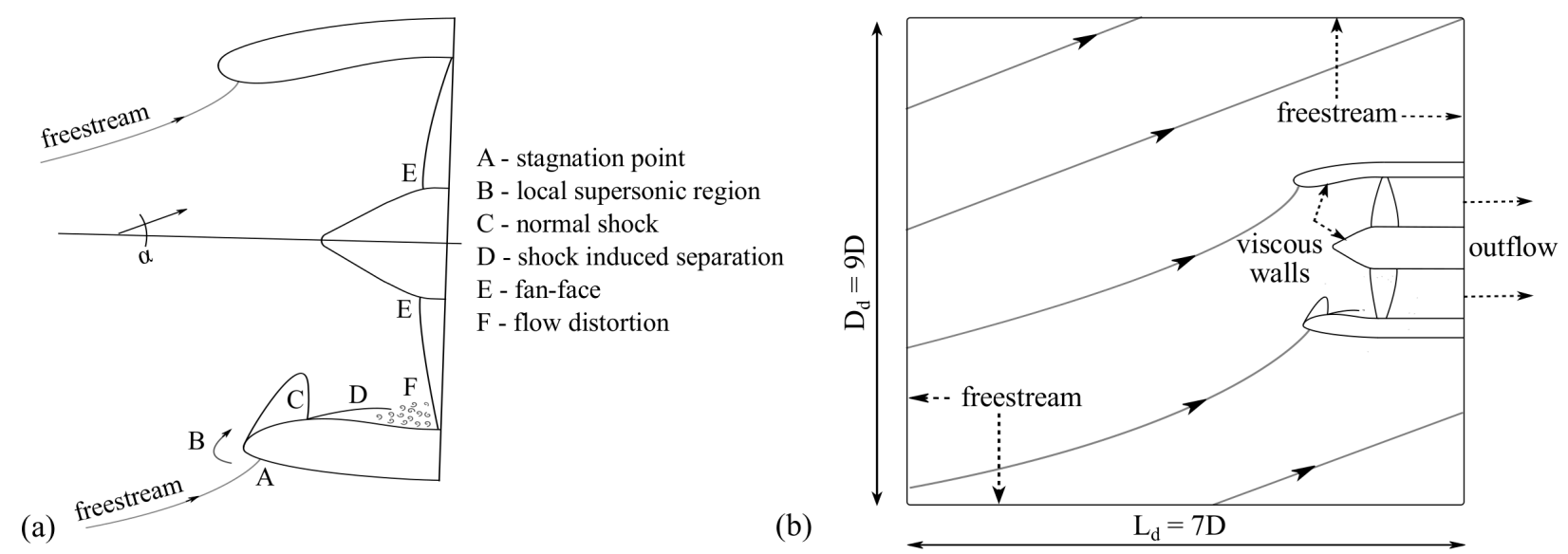

Fig. 1. (a) Key flow features of intake operating under high incidence (b) Computational setup and boundary conditions
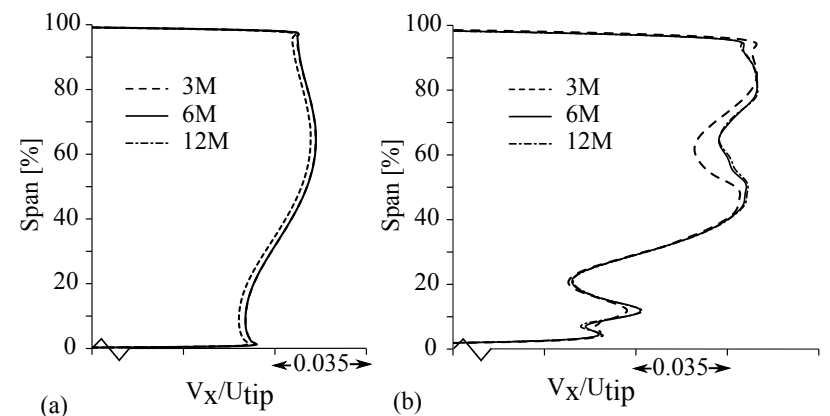

(a)

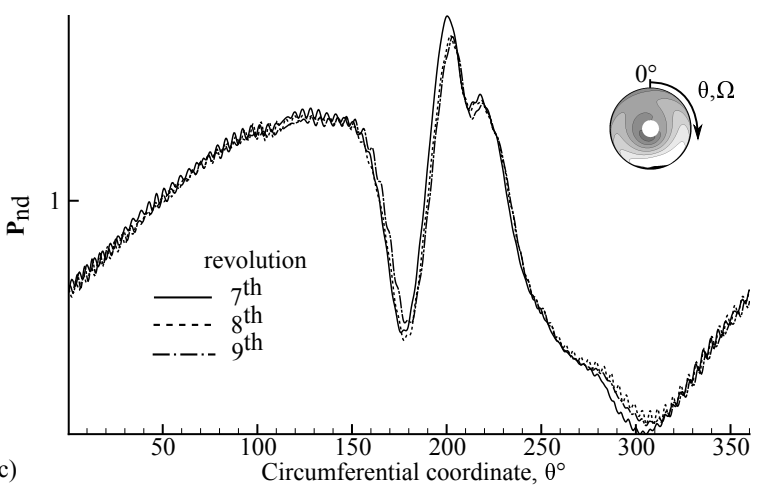

Fig. 2. Validation of characteristic maps: (a) total pressure ratio (b) efficiency and radial profiles: (c) stagnation pressure (d) stagnation temperature against measurements at selected operating conditions. 

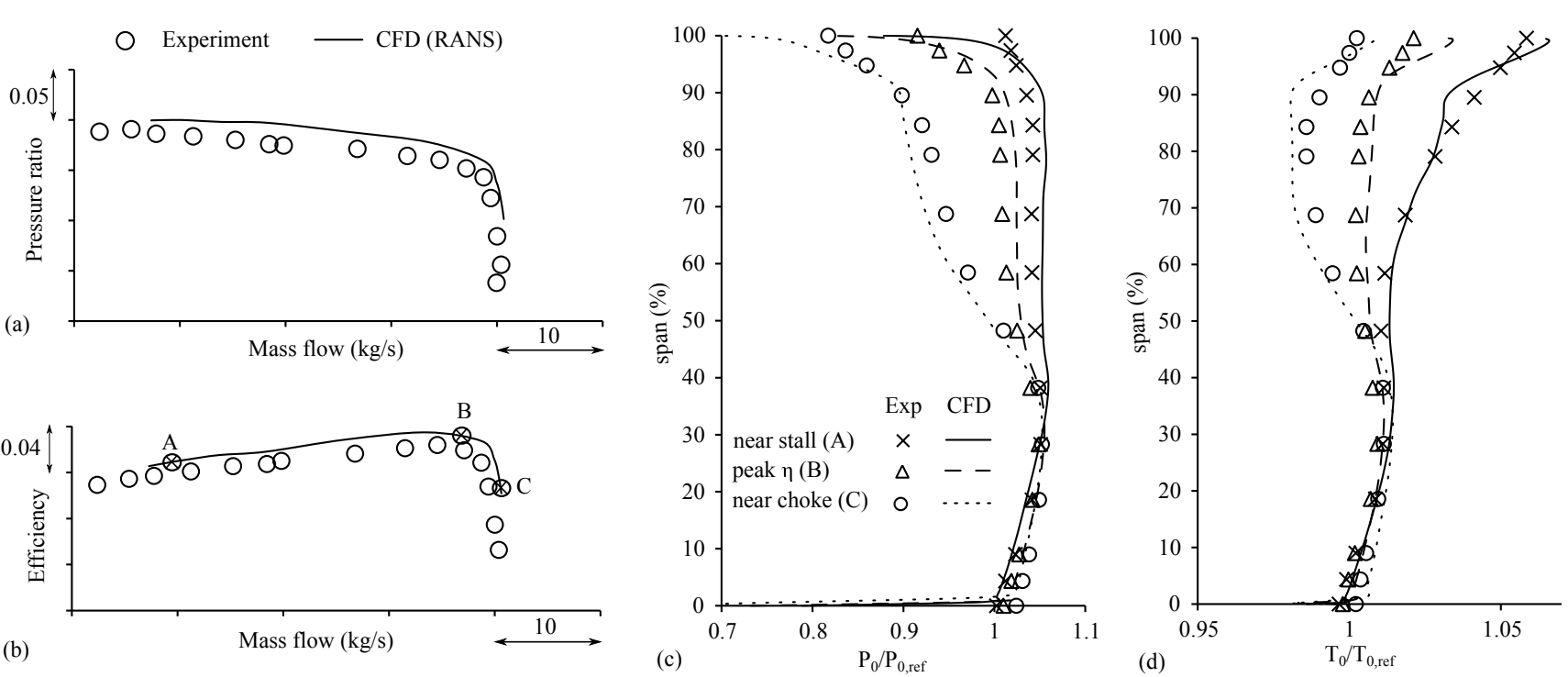

(b)

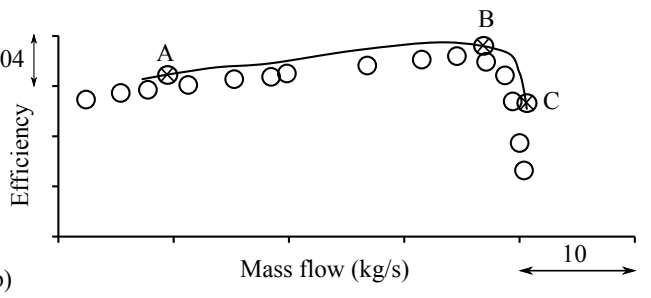

(c)

(d)

Fig. 3. Mesh sensitivity study showing radial traverses of axial velocity non-dimensionalized with the tip speed, (a) upstream and (b) downstream of the rotor. (c) Circumferential variation of power, $\mathbf{P}_{n d}$, on a fan-blade for 3 different revolutions to demonstrate convergence (using 6M mesh per rotor passage).
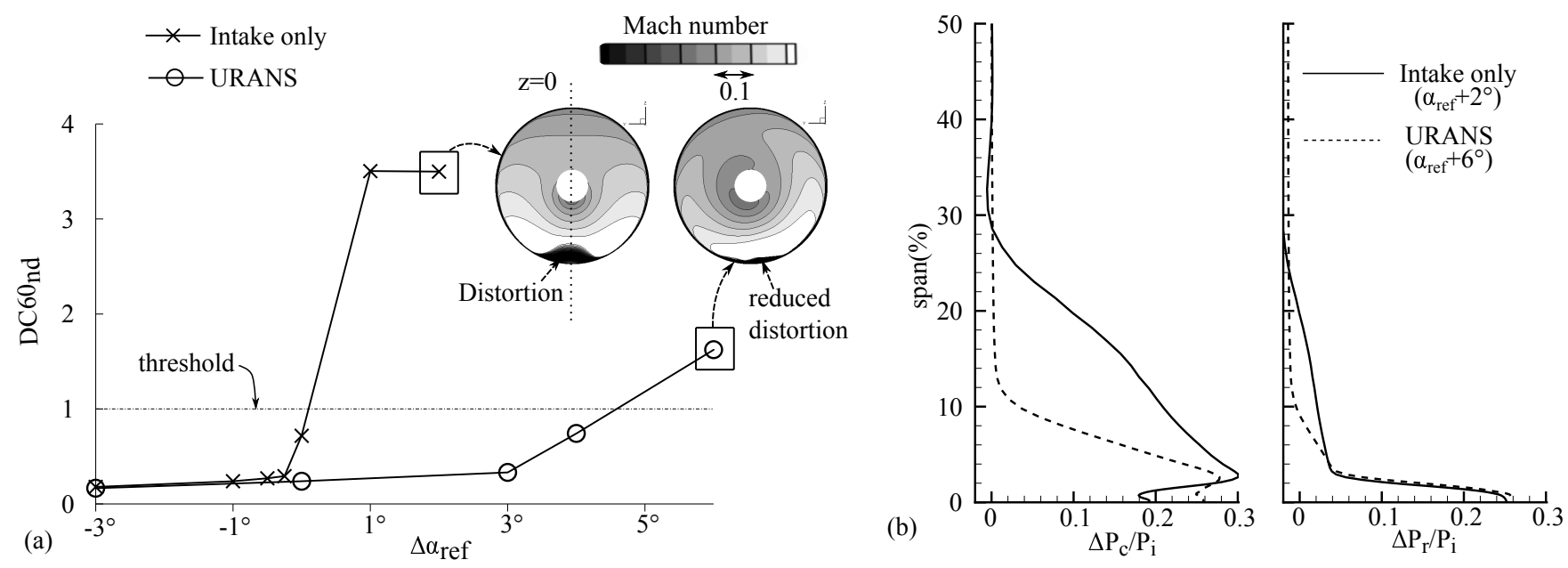

Fig. 4. (a) Variation of normalized distortion coefficient, $D C 60_{n d}$ with increasing incidence for intake-only and intake-fan configurations. Inset plots show the corresponding contours of Mach number at the fan-face (b) Spanwise variation of ARP-1420 quantifying circumferential and radial distortion. 


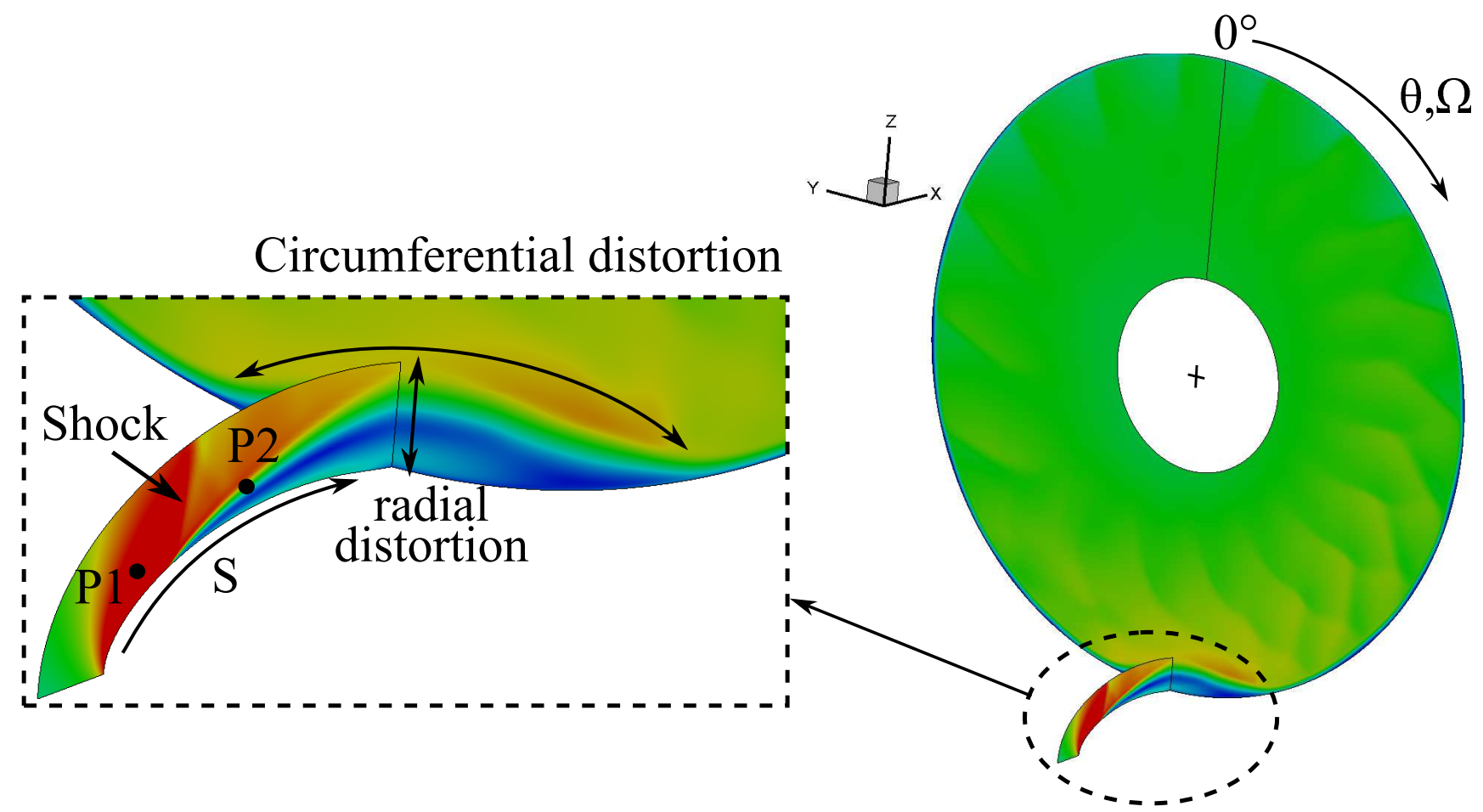

Fig. 5. Instantaneous Mach number distribution on $y=0$ plane and fan-face for $\alpha+6^{\circ}$. Inset plot shows the magnified view of shockinduced separation and subsequent distortion.
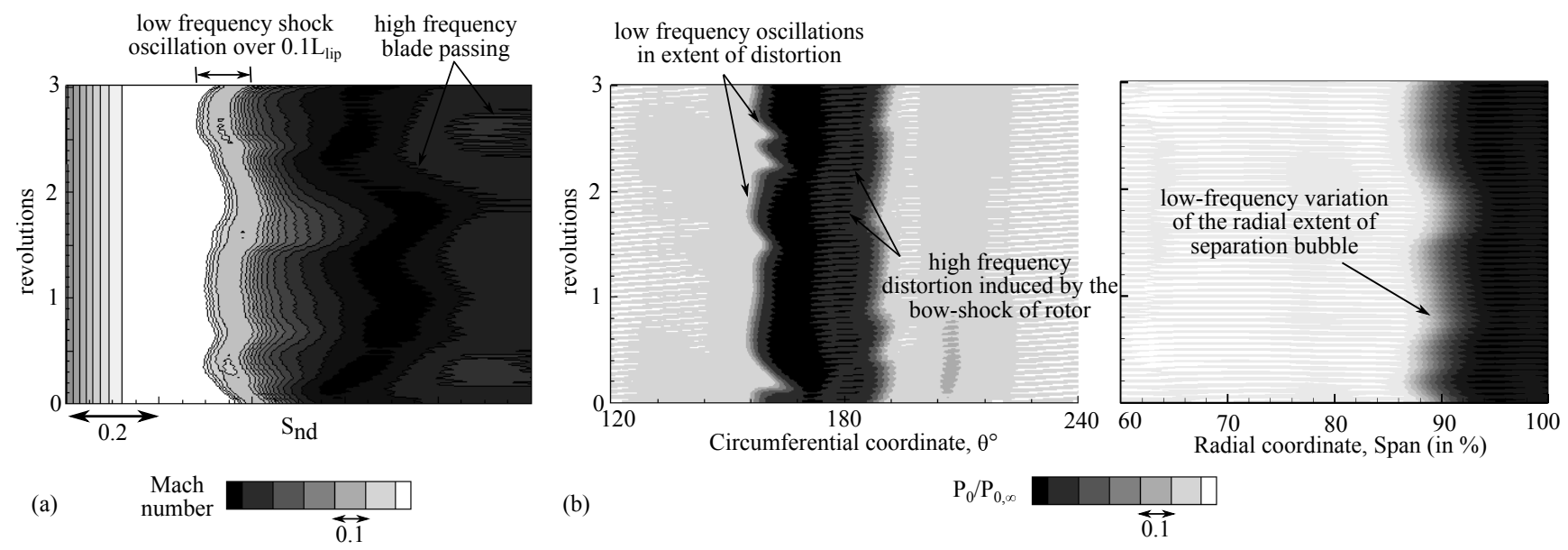

Fig. 6. Space-Time plot of variation of (a) Mach-number along the intake lip on $y=0$ plane (b) stagnation pressure along the circumference and (c) stagnation pressure along the radial direction for three rotor revolutions 


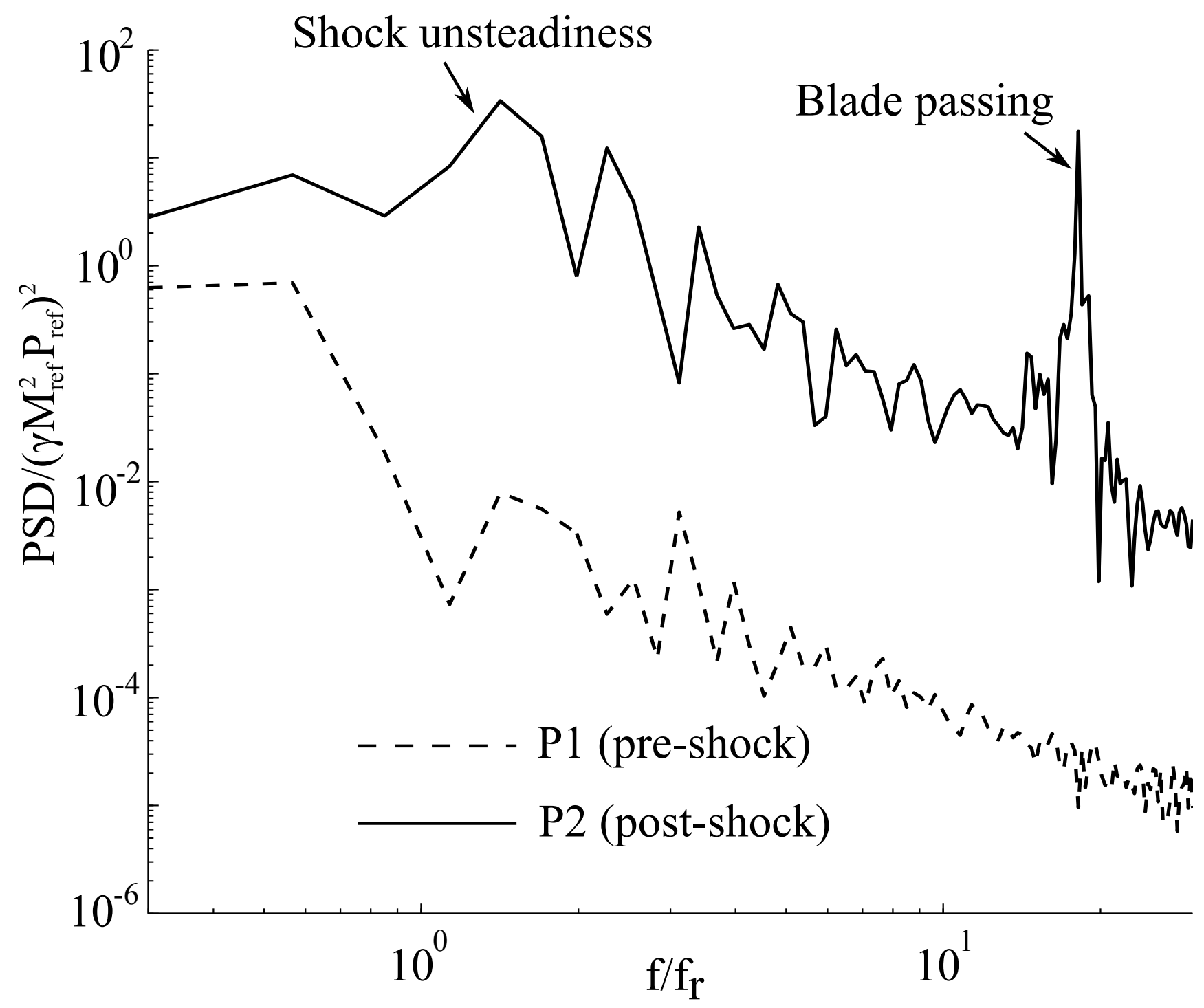

Fig. 7. Power Spectral Density (PSD) of pressure fluctuations measured at probes P1 (pre-shock) and P2 (post-shock) 


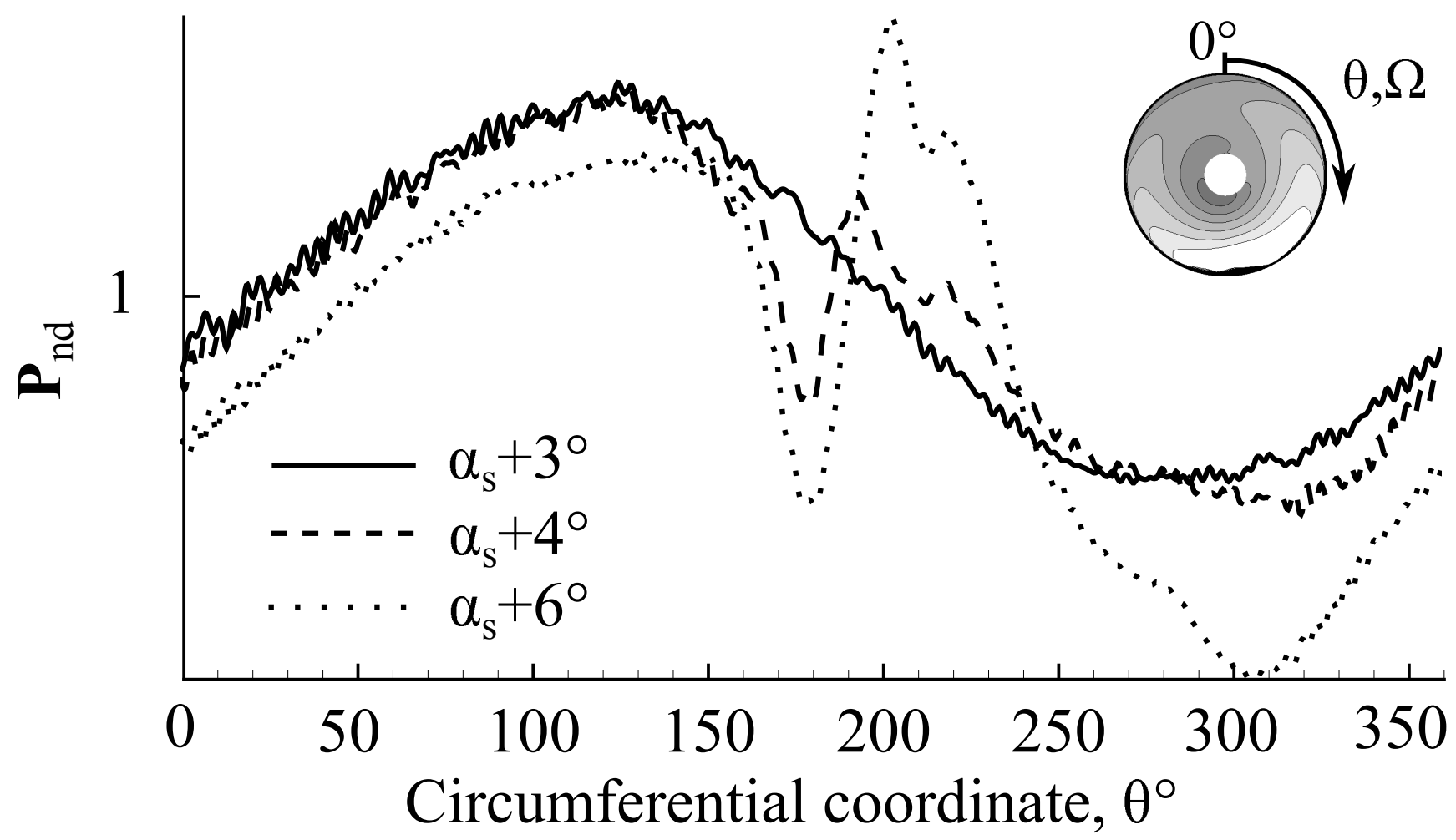

Fig. 8. Circumferential variation of non-dimensional power, $\mathbf{P}_{n d}$, on a fan blade for AoA's: $\alpha+3^{\circ}, \alpha+4^{\circ}, \alpha+6^{\circ}$ 

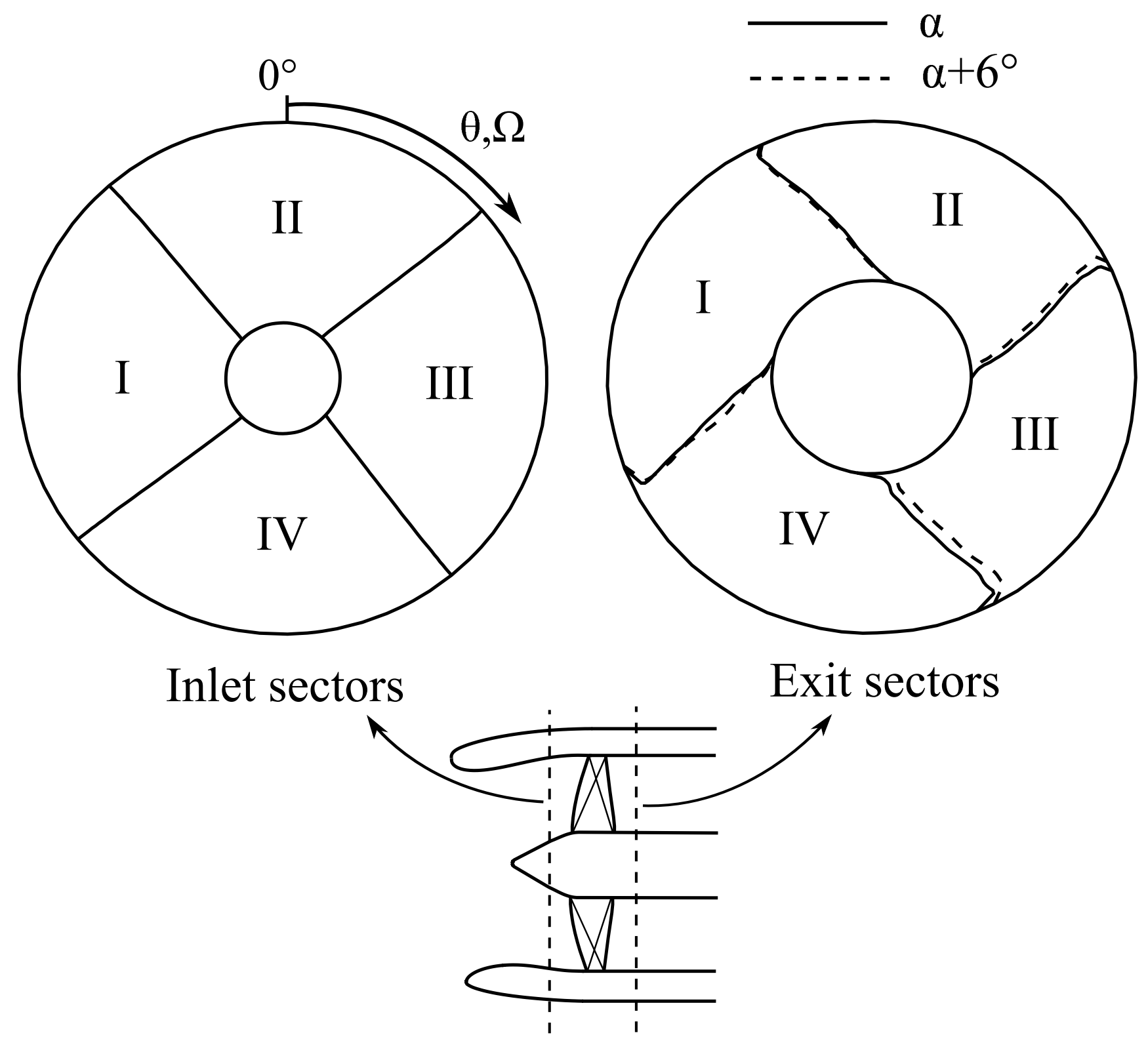

Fig. 9. Inlet and exit sectors used to estimate local operating condition of the rotor 


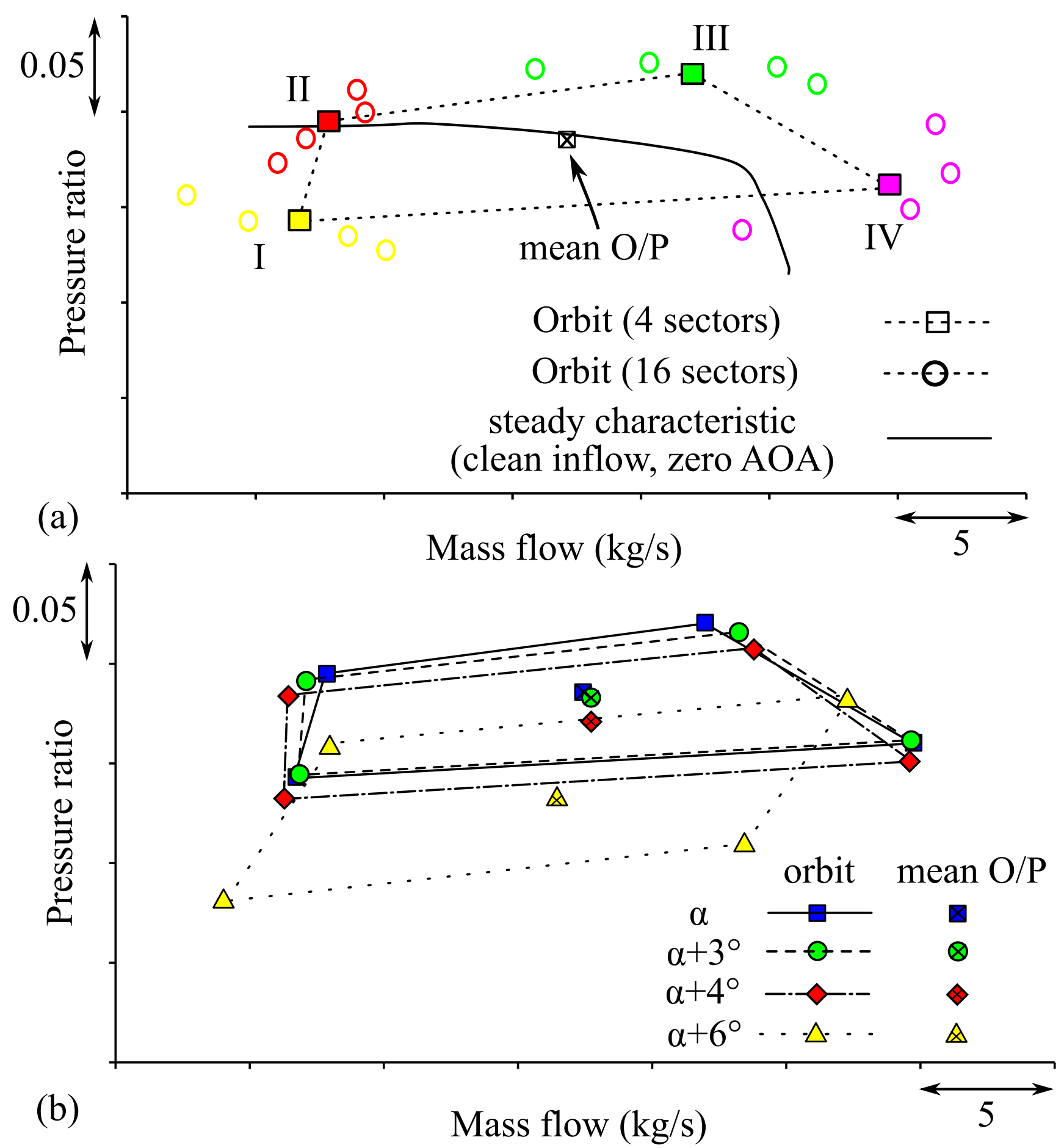

Fig. 10. Local operating condition of the rotor, relative to mean point, when flow over intake lip is (a) attached $\left(A \circ A=\alpha^{\circ}\right)$ and $(b)$ separated $\left(A \circ A=\alpha+6^{\circ}\right)$ 

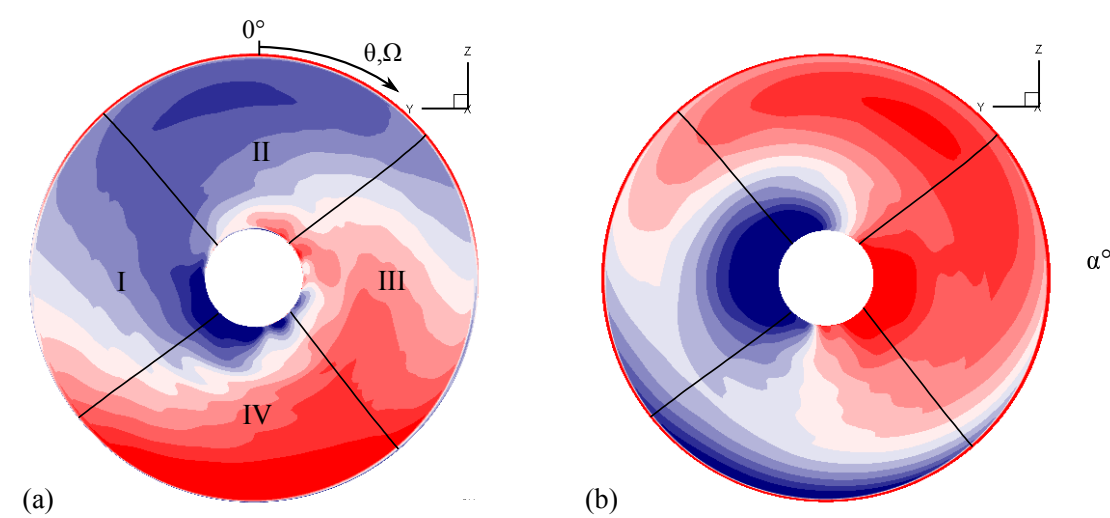

(c)

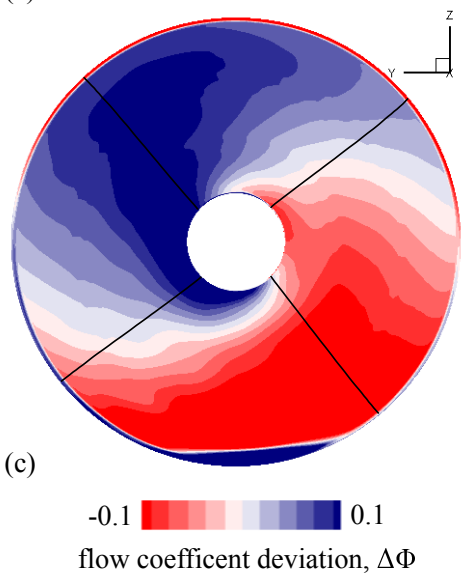

(b)

(d)

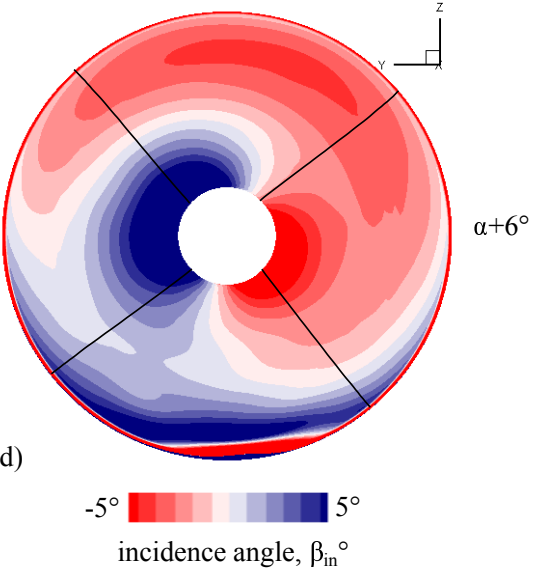

Fig. 11. Contours of (a,c) flow coefficient deviation, $\Delta \Phi$ and (b,d) incidence angle, $\beta_{i n}^{\circ}$ for AoA's of $\alpha$ and $\alpha+6^{\circ}$ 


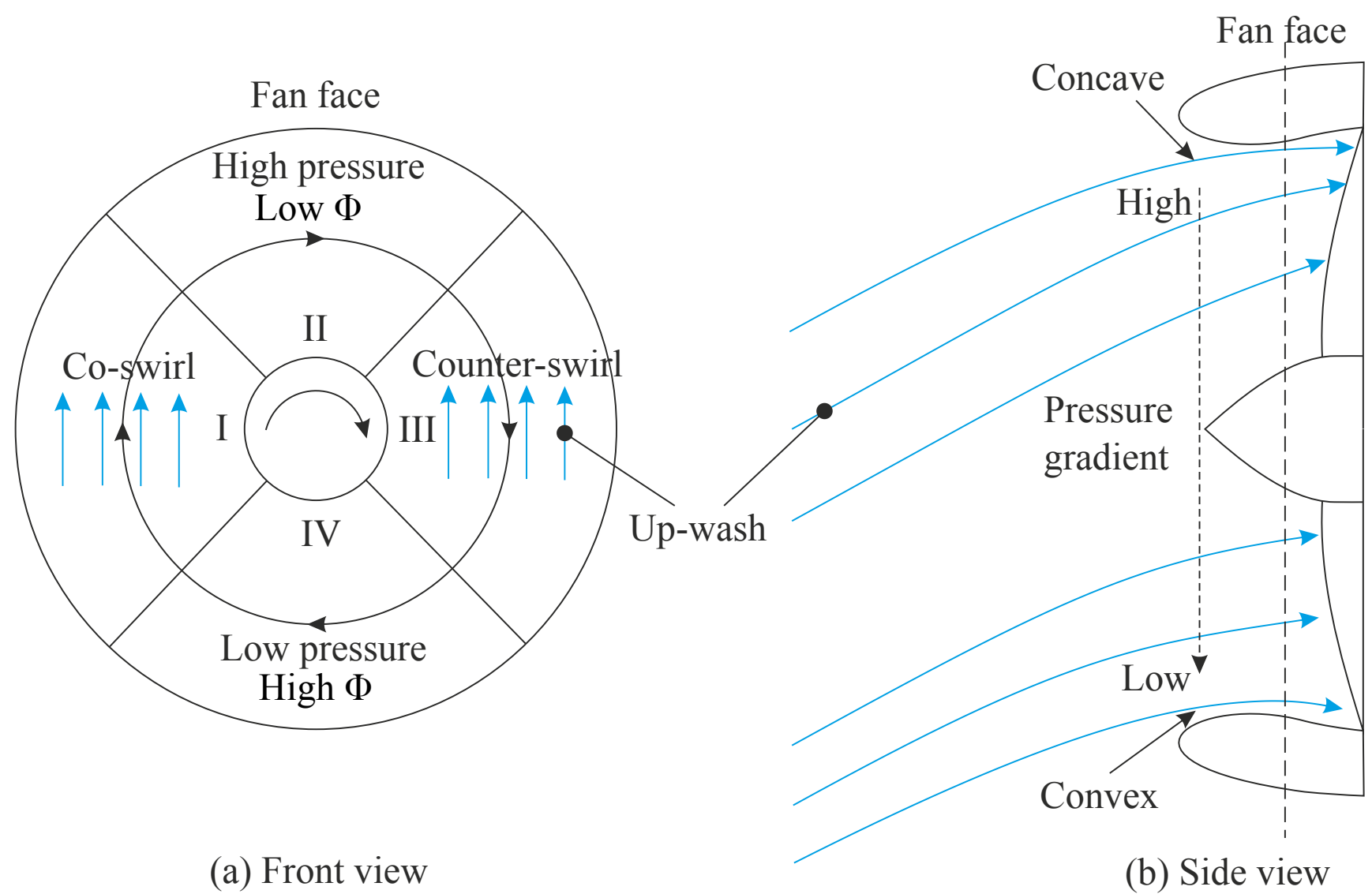

Fig. 12. Sketch illustrating streamline curvature effect and upwash effect

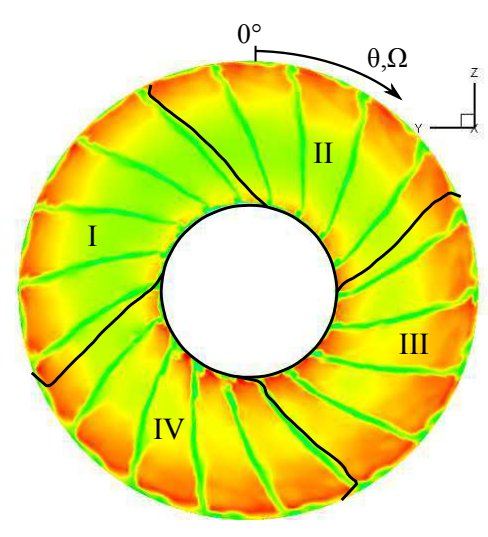

$\alpha$ $\stackrel{0.1}{\leftrightarrow}$

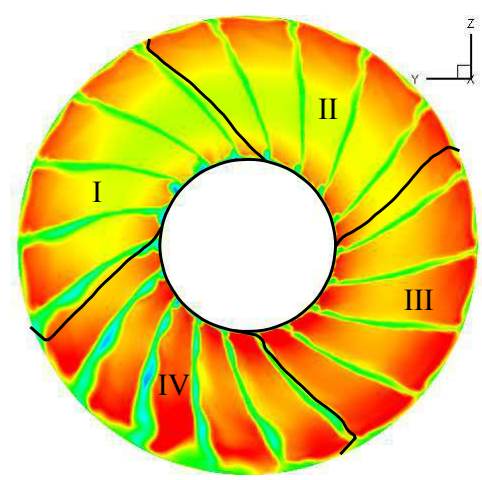

$\alpha+6^{\circ}$

Mach number

Fig. 13. Instantaneous wakes shown through the contours of relative Mach number downstream of the fan at AoA's of $\alpha$ and $\alpha+6^{\circ}$. Exit sectors are marked with solid lines for clarity. 

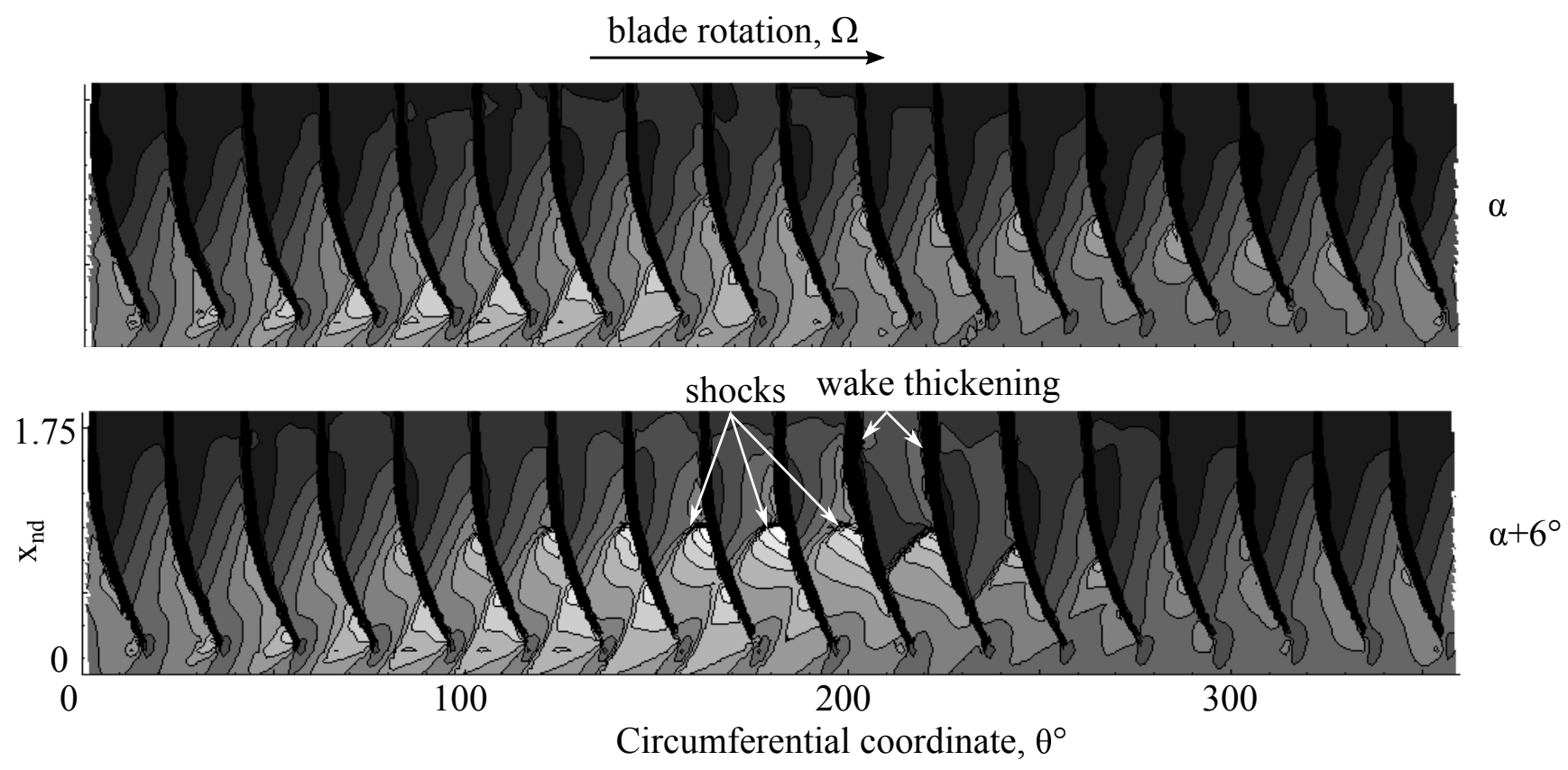

Fig. 14. Unwrapped blade-to-blade snapshots showing contours of relative Mach number at mid-span for AoA's of $\alpha$ and $\alpha+6^{\circ}$

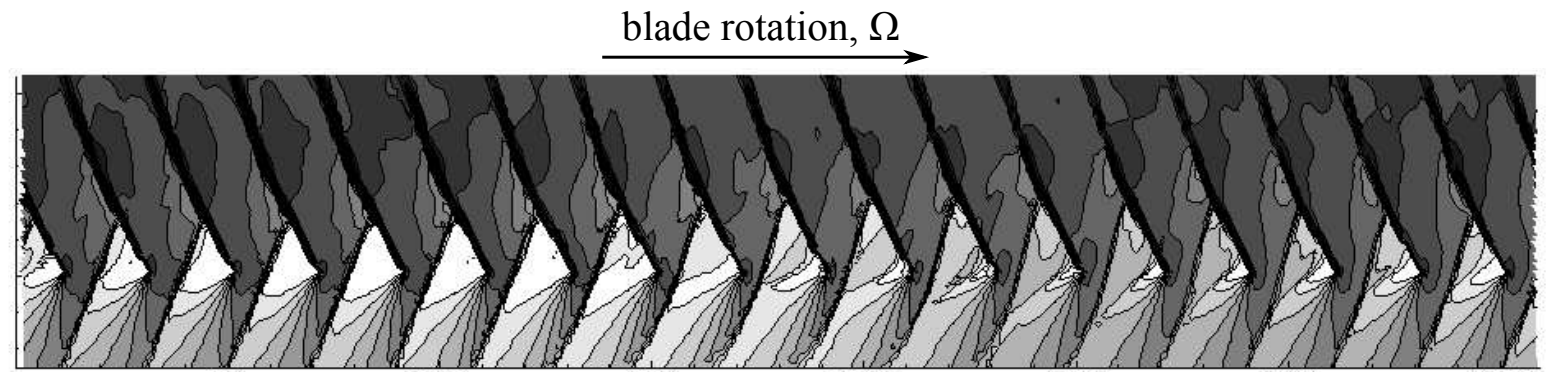

\section{Distortion transfer}

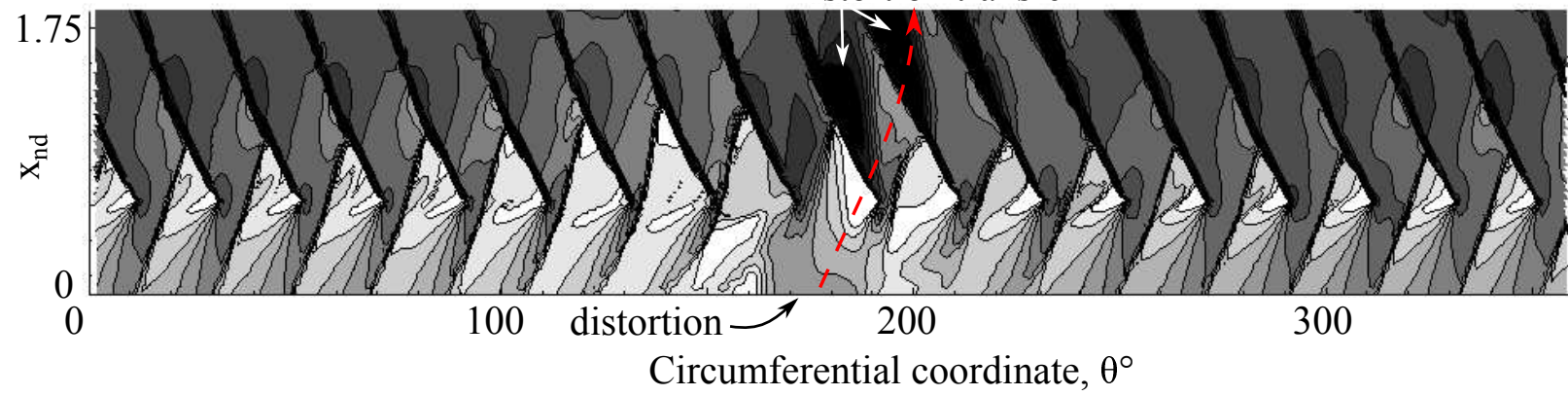

Fig. 15. Unwrapped blade-to-blade snapshots showing contours of relative Mach number at $90 \%$ span for AoA's of $\alpha$ and $\alpha+6^{\circ}$ 